\title{
The escape problem for mortal walkers
}

\author{
D. S. Grebenkov, ${ }^{1,}$ and J.-F. Rupprecht ${ }^{2}$ \\ 1 Laboratoire de Physique de la Matière Condensée (UMR 7643), \\ CNRS - Ecole Polytechnique, University Paris-Saclay, 91128 Palaiseau, France \\ 2 Mechanobiology Institute, National University of Singapore, \\ 5 A Engineering Drive 1, 117411, Singapore
}

(Dated: October 6, 2018)

\begin{abstract}
We introduce and investigate the escape problem for random walkers that may eventually die, decay, bleach, or lose activity during their diffusion towards an escape or reactive region on the boundary of a confining domain. In the case of a first-order kinetics (i.e., exponentially distributed lifetimes), we study the effect of the associated death rate onto the survival probability, the exit probability, and the mean first passage time. We derive the upper and lower bounds and some approximations for these quantities. We reveal three asymptotic regimes of small, intermediate and large death rates. General estimates and asymptotics are compared to several explicit solutions for simple domains, and to numerical simulations. These results allow one to account for stochastic photobleaching of fluorescent tracers in bio-imaging, degradation of mRNA molecules in genetic translation mechanisms, or high mortality rates of spermatozoa in the fertilization process. This is also a mathematical ground for optimizing storage containers and materials to reduce the risk of leakage of dangerous chemicals or nuclear wastes.
\end{abstract}

PACS numbers: 02.50.-r, 05.40.-a, 02.70.Rr, 05.10.Gg

Keywords: leakage, safety, diffusion, escape problem, first passage time, mixed boundary condition

\section{INTRODUCTION}

The safe long-term storage of dangerous species is of paramount importance for chemical industries, nuclear waste containers and landfills, and military arsenals. In spite of numerous efforts to improve containers for dangerous chemicals or nuclear wastes, a complete isolation is not realistic because of eventual defects and material degradation in time. If the species remain active forever, their leakage is certain and is just a matter of time. In such situation, the quality of isolation can be characterized by the survival probability for diffusing species to remain inside a confining domain up to time $t$. This is an example of the first passage time (FPT) problems that have attracted much attention during the last decade [1[4], with numerous chemical [5], biological [6 9] and ecological applications ranging from diffusion in cellular microdomains [10] to animal foraging strategies [11]. Most analytical results were obtained for the mean first passage time (MFPT) through a small escape region, also known as the narrow escape problem [4, 12 18].

In this paper, we introduce and discuss an important extension of the escape problem to "mortal" walkers. In fact, a finite lifetime of diffusing species is a typical situation for many biological, chemical and ecological processes: (i) an animal or a bacterium should remain alive while searching for food; (ii) in bio-imaging techniques, progressive extinction of the fluorescence signal can be either due to degradation of the tagged protein through an enzymatic reaction (i.e. finding the target)

*Electronic address: denis.grebenkov@polytechnique.edu or due to bleaching (i.e. finite fluorescence lifetime); the latter mechanism should be taken into account for reliable interpretation of such measurements; (iii) in order to trigger translation, messenger RNA should not be degradated before reaching a ribosome [19]; (iv) in spite of a very high mortality rate, the spermatozoa that search for a small egg in the uterus or in the Fallopian tubes, need to remain alive to complete the fertilization [20 22]; (v) molecules should remain active or intact before reaching a reactive site on the surface of a catalyst; (vi) protective materials may trap, bind or deactivate dangerous species via a bulk reaction before they leak through defects in the boundary of the container; (vii) for a safe storage of nuclear wastes, the motion of radioactive nuclei should be slowed down enough to ensure their disintegration or at least to reduce the amount of released nuclei, etc. While some first passage problems have been recently extended to mortal walkers (see 22 27] and references therein), the effect of a finite lifetime of a walker onto the escape through the boundary of two- and three-dimensional confining domains has not been investigated. Since the escape is not certain, because of a possible "death" of the walker, the contribution of long trajectories towards the escape region can be greatly reduced, thus strongly affecting the conventional results.

The paper is organized as follows. In Sec. II. we formulate the general escape problem for mortal walkers and we then discuss the most relevant case of a first-order bulk kinetics. We also introduce the exit probability of mortal walkers. In Sec. III we quantify the impact of the finite lifetime of the walkers onto their survival probability, MFPT, and exit probability. For this purpose, we derive the upper and lower bounds of the Laplace-transformed survival probability and related quantities for arbitrary 
bounded domains, and analyze their asymptotic behavior at small, intermediate, and large death rates. To check the quality of these general estimates and asymptotics, we compare them to exact solutions that we obtain for concentric domains and for an escape region on the boundary of a disk. In Sec. IV, various extensions and applications of these results are discussed, in particular, the problem of leakage control and optimization. Section V summarizes and concludes the paper.

\section{NOTATIONS AND EQUATIONS}

In mathematical terms, the first passage time $\tau$ is a random variable which is determined by the survival probability $S\left(t ; x_{0}\right)=\mathbb{P}_{x_{0}}\{\tau>t\}$ that a particle started at a point $x_{0} \in \Omega$ has not left a confining domain $\Omega \subset \mathbb{R}^{d}$ through an escape region $\Gamma$ on the boundary $\partial \Omega$. The survival probability can be expressed through the diffusion propagator for the escape problem, $G_{t}\left(x ; x_{0}\right)$, i.e., the probability density for a particle started at $x_{0} \in \Omega$, to be at position $x$ after time $t$. For conventional immortal walkers, the propagator satisfies the diffusion equation subject to mixed Dirichlet-Neumann boundary condition:

$$
\begin{aligned}
\frac{\partial}{\partial t} G_{t}\left(x ; x_{0}\right)-D \Delta G_{t}\left(x ; x_{0}\right) & =0 \quad(x \in \Omega), \\
G_{t=0}\left(x ; x_{0}\right) & =\delta\left(x-x_{0}\right) \quad(x \in \Omega), \\
\frac{\partial}{\partial n} G_{t}\left(x ; x_{0}\right) & =0 \quad(x \in \partial \Omega \backslash \Gamma), \\
G_{t}\left(x ; x_{0}\right) & =0 \quad(x \in \Gamma),
\end{aligned}
$$

where $D$ is the diffusion coefficient, $\Delta$ the Laplace operator, $\delta\left(x-x_{0}\right)$ is the Dirac distribution, and $\partial / \partial n$ the normal derivative [1, 28]. The survival probability is then

$$
S\left(t ; x_{0}\right)=\int_{\Omega} d x G_{t}\left(x ; x_{0}\right) .
$$

The Laplace transform reduces the diffusion equation for the survival probability (which follows from Eq. (10) to a simpler Helmholtz equation,

$$
[D \Delta-p] \tilde{S}\left(p ; x_{0}\right)=-1,
$$

with the same mixed boundary condition, where tilde denotes the Laplace-transformed survival probability:

$$
\tilde{S}\left(p ; x_{0}\right)=\int_{0}^{\infty} d t e^{-t p} S\left(t, x_{0}\right) .
$$

Once Eq. (3) is solved, the inverse Laplace transform of $\tilde{S}\left(p ; x_{0}\right)$ yields the survival probability $S\left(t ; x_{0}\right)$ in time domain. Note that the probability density of the FPT is

$$
\rho\left(t ; x_{0}\right)=-\frac{\partial}{\partial t} S\left(t ; x_{0}\right),
$$

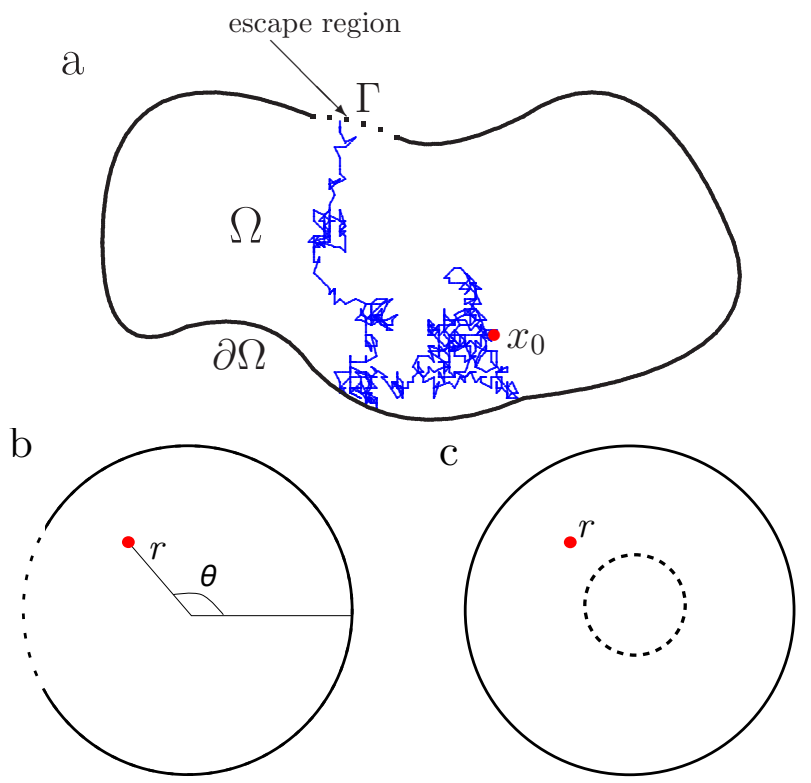

FIG. 1: (Color online) Schematic illustration of the escape problem. (a) A general domain $\Omega \subset \mathbb{R}^{d}$ with an escape region $\Gamma$ (dashed line) on its boundary $\partial \Omega$. A particle is released at a starting point $x_{0}$ and diffuses, with eventual reflections on $\partial \Omega \backslash \Gamma$, until it reaches the escape region $\Gamma$. (b,c) Two explicitly solvable cases of the escape problem: from a disk through an arc on its boundary (b), see Appendix C] and from an annulus through its inner circle (c), see Appendix A

while the mean FPT is simply $\tilde{S}\left(0 ; x_{0}\right)$ (in particular, setting $p=0$ in Eq. (3) yields the usual Poisson equation for the MFPT [1]).

Next, we formulate the escape problem for mortal walkers. We assume that the lifetime $\chi$ of a walker is independent of the search process. This situation corresponds to the case of a uniform bulk reaction or to some internal death mechanism of a walker, e.g., a life cycle of a bacterium or a radioactive decay. In this case, the survival probability of a mortal walker inside the domain $\Omega$ up to time $t, S_{\mu}\left(t ; x_{0}\right)$, is simply the product of $S\left(t ; x_{0}\right)$ and $Q(t)=\mathbb{P}\{\chi>t\}$ :

$$
S_{\mu}\left(t ; x_{0}\right)=\mathbb{P}\{\min \{\tau, \chi\}>t\}=S\left(t ; x_{0}\right) Q(t) .
$$

While this expression is general, it remains formal as the survival probability $S\left(t ; x_{0}\right)$ is not known analytically except for some elementary cases (see Appendix A).

A particular simplification occurs in the relevant case of an exponentially distributed lifetime, $Q(t)=$ $\exp (-\mu t)$, with $\mu$ being the death rate (or $1 / \mu$ being the mean lifetime), for which the Laplace transform of Eq. (6) reads

$$
\tilde{S}_{\mu}\left(p ; x_{0}\right)=\tilde{S}\left(p+\mu ; x_{0}\right) .
$$

This is equivalent to adding the term $-\mu \tilde{S}$ into Eq. (3) to describe a first-order bulk kinetics or radioactive decay. 
The MFPT for mortal walkers is then

$$
\left\langle\tau_{\mu}\right\rangle=\tilde{S}_{\mu}\left(0 ; x_{0}\right)=\tilde{S}\left(\mu ; x_{0}\right),
$$

where $\langle\cdots\rangle$ denotes the expectation. Since the Laplace transform of a positive function monotonously decreases, the MFPT monotonously decreases with $\mu$. In particular, the MFPT for mortal walkers is smaller than that for immortal ones. This is expected because long trajectories to the escape region are progressively eliminated as $\mu$ increases.

Finally, we introduce the exit probability of mortal walkers, $H_{\mu}\left(x_{0}\right)$, i.e., the probability that a walker started from $x_{0}$ leaves the confining domain before dying: $H_{\mu}\left(x_{0}\right)=\mathbb{P}\{\tau<\chi\}$. Since $\tau$ and $\chi$ are independent, the exit probability can in general be expressed as

$$
\begin{aligned}
H_{\mu}\left(x_{0}\right) & =\int_{0}^{\infty} \mathbb{P}\{\chi>t\} \mathbb{P}\{\tau \in(t, t+d t)\} \\
& =\int_{0}^{\infty} d t Q(t) \rho\left(t ; x_{0}\right) .
\end{aligned}
$$

For an exponentially distributed lifetime, the exit probability becomes

$$
H_{\mu}\left(x_{0}\right)=\int_{0}^{\infty} d t e^{-\mu t} \rho\left(t ; x_{0}\right)=1-\mu \tilde{S}\left(\mu ; x_{0}\right)=1-\mu\left\langle\tau_{\mu}\right\rangle
$$

where we used Eq. (5). The exit probability satisfies

$$
\begin{aligned}
D \Delta H_{\mu}\left(x_{0}\right)-\mu H_{\mu}\left(x_{0}\right) & =0 & & \left(x_{0} \in \Omega\right), \\
H_{\mu}\left(x_{0}\right) & =1 & & \left(x_{0} \in \Gamma\right), \\
\frac{\partial}{\partial n} H_{\mu}\left(x_{0}\right) & =0 & & \left(x_{0} \in \partial \Omega \backslash \Gamma\right) .
\end{aligned}
$$

As expected, this probability is equal to 1 for immortal walkers (i.e., for the conventional escape problem with $\mu=0$ ) and monotonously decreases to 0 as $\mu$ increases. For mortal walkers, the exit probability characterizes the storage safety of a container.

In many applications, the starting point $x_{0}$ is not fixed but randomly distributed over the confining domain. In this case, one considers the global, or volume-averaged MFPT:

$$
\overline{\left\langle\tau_{\mu}\right\rangle}=\frac{1}{|\Omega|} \int_{\Omega} d x_{0}\left\langle\tau_{\mu}\right\rangle
$$

where $|\Omega|$ is the volume of $\Omega$. In analogy, we define the global exit probability (GEP) to characterize the overall safety of the container:

$$
\bar{H}_{\mu}=\frac{1}{|\Omega|} \int_{\Omega} d x_{0} H_{\mu}\left(x_{0}\right)
$$

Integrating Eq. (11) over the confining domain and using the Green formula and boundary conditions, one gets another representation of the GEP:

$$
\bar{H}_{\mu}=\frac{D}{\mu|\Omega|} \int_{\Gamma} d x_{0} \frac{\partial H_{\mu}\left(x_{0}\right)}{\partial n}
$$

that will used to derive its general asymptotic expression in the limit of large death rates.

When the mean lifetime $1 / \mu$ is much larger that the time needed to find the escape region by immortal walkers, i.e., $\mu\left\langle\tau_{0}\right\rangle \ll 1$, the Taylor expansion of Eq. (4) yields

$$
\left\langle\tau_{\mu}\right\rangle=\left\langle\tau_{0}\right\rangle-\frac{1}{2} \mu\left\langle\tau_{0}^{2}\right\rangle+O\left(\mu^{2}\right)
$$

and, from Eq. (10),

$$
H_{\mu}\left(x_{0}\right)=1-\mu\left\langle\tau_{0}\right\rangle+\frac{1}{2} \mu^{2}\left\langle\tau_{0}^{2}\right\rangle+O\left(\mu^{3}\right) .
$$

The problem is reduced to the analysis of the moments of the FPT of immortal walkers

$$
\left\langle\tau_{0}^{n}\right\rangle=(-1)^{n}\left(\frac{\partial^{n}}{\partial \mu^{n}} H_{\mu}\left(x_{0}\right)\right)_{\mu=0} .
$$

In particular, the exact formulas for the MFPT are known for several simple domains [4, 14, 29 31] (see also Appendices $\mathrm{A}$ and $\mathrm{C}$ ). When the escape region is small, the following asymptotic behavior was established (see the review [4] and references therein):

$$
\left\langle\tau_{0}\right\rangle \simeq \begin{cases}\frac{|\Omega|}{\pi D}(\ln (1 / \epsilon)+O(1)) & (d=2) \\ \frac{|\Omega|}{4 D R \epsilon}+O(\ln \epsilon) & (d=3)\end{cases}
$$

where $R=|\partial \Omega|^{1 /(d-1)}$ is the characteristic size of the domain, and $\epsilon=(|\Gamma| /|\partial \Omega|)^{1 /(d-1)}$ is the perimeter of the escape region $\Gamma$ normalized by the perimeter of the boundary $\partial \Omega$ (for $d=2$ ), or the square root of the area of the escape region normalized by the area of the boundary (for $d=3$ ).

In the context of chemical reactions, the escape region can be interpreted as an active catalytic site on an inert confining boundary of a reactor, the $\mathrm{FPT} \tau_{\mu}$ is the (random) reaction time (for a perfectly reactive catalyst), $\left\langle\tau_{\mu}\right\rangle$ is the mean reaction time, and $H_{\mu}\left(x_{0}\right)$ is the target encounter probability. While we adopt the language of first-passage processes, the following results can be easily translated into the chemical or biochemical context.

In what follows, we investigate the Laplacetransformed survival probability, the MFPT, the exit probability and the GEP for mortal walkers in the limit of short lifetimes, $\mu\left\langle\tau_{0}\right\rangle \gg 1$, which turns out to be more relevant for many applications, e.g., for developing safe containers. Note also that the large $\mu$ limit captures the short-time asymptotic behavior of the survival probability and related quantities according to Tauberian theorems. In contrast to the long-time asymptotics, the 
short-time behavior of first passage distributions is generally not universal [32]. Nevertheless, we will obtain a universal relation (28) for the global exit probability within the short-time scale regime.

\section{MAIN RESULTS}

\section{A. Upper and lower bounds}

First, we establish the upper and lower bounds for the Laplace-transformed survival probability. An elementary upper bound follows from the trivial inequality $S\left(t, x_{0}\right) \leq$ 1 by applying the Laplace transform:

$$
\tilde{S}\left(\mu ; x_{0}\right) \leq \frac{1}{\mu} .
$$

The lower bound can be obtained from the continuity of Brownian motion that implies that the FPT to a subset $\Gamma$ of the boundary $\partial \Omega$ is greater than the FPT from the center of the ball of radius $\left|x_{0}-\partial \Omega\right|$ to its boundary, where $\left|x_{0}-\partial \Omega\right|$ is the distance from $x_{0}$ to the boundary $\partial \Omega$. In probabilistic terms, it means that

$$
S\left(t ; x_{0}\right) \geq S_{B_{d}\left(\left|x_{0}-\partial \Omega\right|\right)}(t ; 0),
$$

where $S_{B_{d}(R)}(t ; 0)$ is the survival probability inside a $d$ dimensional ball of radius $R, B_{d}(R)$, for a particle started from the center of that ball. We claim a stronger inequality

$$
S\left(t ; x_{0}\right) \geq S_{B_{d}\left(\left|x_{0}-\Gamma\right|\right)}(t ; 0),
$$

where the distance $\left|x_{0}-\partial \Omega\right|$ to the boundary $\partial \Omega$ is replaced by the distance $\left|x_{0}-\Gamma\right|$ to the escape region $\Gamma$ (see Appendix B). Since the Laplace transform of positive functions does not affect the inequality, one also gets the explicit lower bound in the Laplace domain

$$
\tilde{S}\left(\mu ; x_{0}\right) \geq \tilde{S}_{B_{d}\left(\left|x_{0}-\Gamma\right|\right)}(\mu ; 0) .
$$

The right-hand side is known explicitly:

$$
\tilde{S}_{B_{d}(R)}(\mu ; 0)=\frac{1}{\mu}\left(1-U_{d}(R \sqrt{\mu / D})\right),
$$

with

$$
U_{d}(x)=\frac{(x / 2)^{\frac{d}{2}-1}}{\Gamma\left(\frac{d}{2}\right) I_{\frac{d}{2}-1}(x)},
$$

where $\Gamma(d / 2)$ is the Gamma function, and $I_{\nu}(z)$ is the modified Bessel function of the first kind (see Appendix A 1). When $x \gg 1$, one has

$$
U_{d}(x) \simeq \frac{\sqrt{\pi} 2^{\frac{3-d}{2}}}{\Gamma(d / 2)} x^{\frac{d-1}{2}} e^{-x} .
$$

Combining the upper and lower bounds (19, 22), one concludes that the MFPT, $\left\langle\tau_{\mu}\right\rangle=\tilde{S}\left(\mu ; x_{0}\right)$, can be accurately approximated as $1 / \mu$ at large $\mu$ for any domain
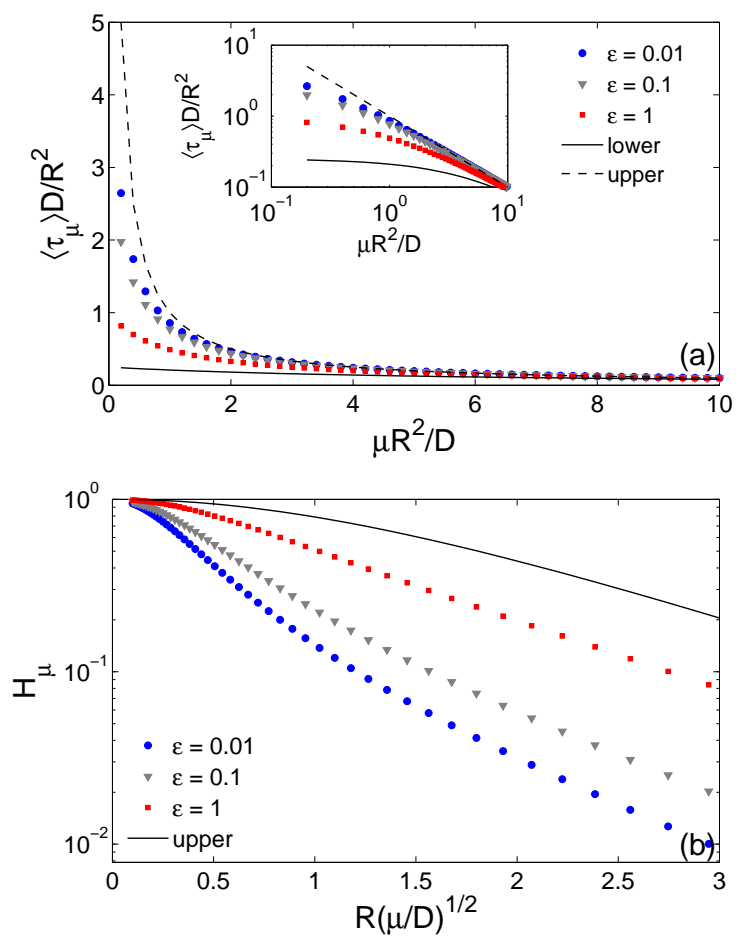

FIG. 2: (Color online) The MFPT $\left\langle\tau_{\mu}\right\rangle$ (a) and the exit probability $H_{\mu}\left(x_{0}\right)$ (b), as functions of $\mu R^{2} / D$ and $R \sqrt{\mu / D}$, respectively, for the disk of radius $R$ with the escape arc $(\pi-$ $\varepsilon, \pi+\varepsilon$ ) on the boundary, for several $\varepsilon$ (here $x_{0}=0$ ). Inset shows the MFPT on log-log scale. For the MFPT, the exact solution C1 is compared to the upper and lower bounds (19) 22). For the exit probability, the exact solution (C10) is compared to the upper bound (26) (the lower bound being zero).

and any escape region, the error of this approximation vanishing as a stretched-exponential function according to Eq. (25). As a consequence, the MFPT becomes fully controlled by the lifetime of the walker in this limit, as expected. Similarly, the global MFPT, $\overline{\left\langle\tau_{\mu}\right\rangle}$, behaves as $1 / \mu$ at large $\mu$, although it includes contribution from points which are close to the escape region.

Figure 2a illustrates this behavior in the case of a disk with an escape region on its boundary, for which the exact formula for the Laplace-transformed survival probability was recently derived [30] (see Appendix C). One can see that the two bounds accurately approximate the MFPT at large $\mu$. In turn, they do not control the behavior of the MFPT at small $\mu$, in which case the lower bound tends to $\left|x_{0}-\Gamma\right|^{2} /(2 d D)$, and the upper bound diverges. In this limit, one can use the asymptotic relation (15).

In turn, the exit probability $H_{\mu}\left(x_{0}\right)$ becomes a nontrivial characteristics of the escape problem at large $\mu$. According to Eqs. (10, 22), $H_{\mu}\left(x_{0}\right)$ is bounded as

$$
H_{\mu}\left(x_{0}\right) \leq U_{d}\left(\left|x_{0}-\Gamma\right| \sqrt{\mu / D}\right) .
$$


Since the upper bound is independent of the size of the escape region, it does not capture well the accessibility of this region. In fact, the exit probability is expected to decay faster with $\mu$ for smaller escape regions. This is illustrated in Fig. 2 $\mathrm{b}$ that shows $H_{\mu}\left(x_{0}\right)$ for the disk with escape regions of various sizes. While the upper bound over-estimates the exit probability, it captures correctly its asymptotic decay as a stretched-exponential function. The quality of the upper bound (26) is further discussed in the case of a disk in Appendix C3. Note also that this upper bound is equal to the exit probability in the case of a ball with the escape region on the whole boundary and the starting point at the origin.

According to the inequality (26) and numerical analysis, the exit probability $H_{\mu}\left(x_{0}\right)$ at large $\mu$ exponentially decays with the distance from the starting point to the escape region. For a better control of this decay, one needs a lower bound for the exit probability. In Appendix D we partly solve this problem for the specific case when the escape region is the whole boundary: $\Gamma=\partial \Omega$. Finding an appropriate lower bound for the general escape problem (with $\Gamma \neq \partial \Omega$ ) remains an open problem.

\section{B. Global exit probability}

When the starting point is uniformly distributed, some walkers start near the escape region, and the stretchedexponential decay with $\mu$ is expected to be replaced by a slower power law. Determining an exact expression for the GEP appears to be a challenging problem because of mixed boundary conditions. Here, we derive an approximation for the GEP for intermediate death rates, which also turns out to be exact in the limit of small death rates. For small escape regions, this approximation shows satisfactory agreement with the results of numerical simulations over a broad range of $\mu$.

The survival probability $S\left(t ; x_{0}\right)$ in a bounded domain admits a spectral decomposition on appropriate Laplacian eigenfunctions and thus decreases exponentially at long times as $S\left(t ; x_{0}\right) \sim A\left(x_{0}\right) e^{-\lambda t}$, where $\lambda$ is the smallest Laplacian eigenvalue, and $A\left(x_{0}\right)$ is a coefficient. When the escape region is small as compared to the size of the confining domain, one expects $A\left(x_{0}\right) \simeq 1$ and $\overline{\left\langle\tau_{0}\right\rangle}=1 / \lambda$, independently of the starting point $x_{0}$ if $x_{0}$ is far from the escape region [12, 33, 34]. As a consequence, the volume-averaged survival probability behaves as $\bar{S}(t) \sim e^{-\lambda t}$ in this narrow escape limit, from which the Laplace transform yields

$$
\bar{H}_{\mu}=1-\mu \overline{\tilde{S}}(\mu) \approx \frac{1}{1+\mu \overline{\left\langle\tau_{0}\right\rangle}} .
$$

In the limit $\mu \rightarrow 0$, one retrieves $\bar{H}_{0}=1$ for any escape region size, independently of its smallness, as expected. In turn, the exit probability vanishes for any $\mu>0$ in the limit of shrinking escape region because $\overline{\left\langle\tau_{0}\right\rangle} \rightarrow \infty$. Note also that this expression can be re-written as $1 / \overline{\left\langle\tau_{\mu}\right\rangle}=$

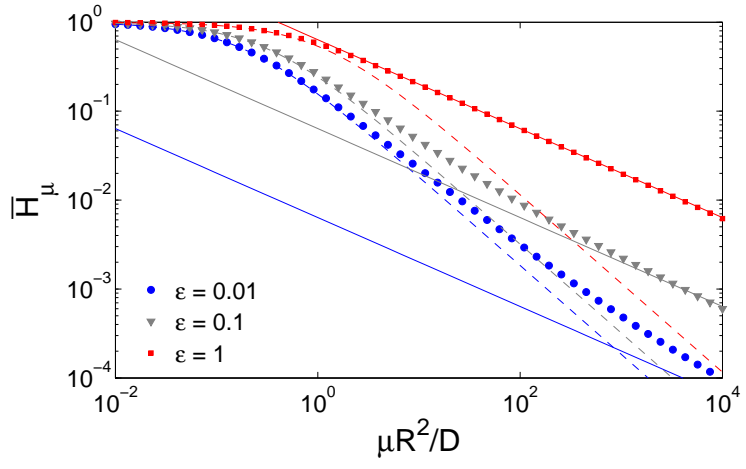

FIG. 3: (Color online) The global (or volume-averaged) exit probability $\bar{H}_{\mu}$ (symbols) from Eq. (C13) as a function of $\mu R^{2} / D$ for the disk of radius $R$ with the escape arc $(\pi-\varepsilon, \pi+\varepsilon)$ on the boundary. Dashed lines show the approximation (27), with the volume-averaged MFPT $\overline{\left\langle\tau_{0}\right\rangle}=\frac{R^{2}}{D}(1 / 8-\ln (\sin (\varepsilon / 2)))[14]$ while solid lines present the asymptotic relation (28).

$1 \overline{\left\langle\tau_{0}\right\rangle}+\mu$, i.e., the volume-averaged MFPT for mortal walkers is the harmonic mean of $\overline{\left\langle\tau_{0}\right\rangle}$ and $1 / \mu$. We point out that Eq. (27) is exact, to the first order, in the limit $\mu \overline{\left\langle\tau_{0}\right\rangle} \ll 1$. This can be seen by comparing the asymptotic expansion of Eq. (27) to the integral over $x_{0}$ of the Taylor expansion (16).

We compare the approximation (27) to two exact solutions: the concentric escape region (Appendix $\mathrm{A}$ ) and the disk with an escape arc $(\pi-\varepsilon, \pi+\varepsilon)$ on the boundary (Appendix C). We present the results only for the latter case, for which Fig. 3 shows the GEP as a function of the death rate. The approximation (27) (shown by dashed lines) accurately captures the behavior of the GEP at small $\mu$ and under-estimates its values at larger $\mu$. In the narrow escape configuration, when $\overline{\left\langle\tau_{0}\right\rangle} \gg R^{2} / D$, the approximation (27) remains accurate even for $\mu \sim 1 \overline{\left\langle\tau_{0}\right\rangle}$. However, Eq. (27) fails at large death rates $\mu \gg D /(\varepsilon R)^{2}$ that correspond to the short-time behavior of the FPT distribution.

The mono-exponential approximation of the survival probability fails at very short times. Similarly to [32], we show that in the large death rate limit (which corresponds to the few-encounter regime in [32]), the notion of a kinetic rate in the traditional bulk sense does not hold, and the asymptotic regime of the GEP should be different. In this limit, the exit probability $H_{\mu}\left(x_{0}\right)$ rapidly decays from the escape region $\Gamma$ towards the bulk. In the vicinity of each escape point, the exit probability along normal vector can be approximated by the onedimensional solution of Eq. (11) on the positive half-line: $H_{\mu}\left(x_{0}\right)=\exp \left(-x_{0} \sqrt{\mu / D}\right)$. Substituting this approximation in Eq. (14), one finds

$$
\bar{H}_{\mu} \underset{\mu \rightarrow \infty}{\sim} \frac{|\Gamma|}{|\Omega|}(\mu / D)^{-\frac{1}{2}} .
$$


The right-hand side can be interpreted as the probability that a uniformly distributed starting point lies within a thin layer of width $\ell=(\mu / D)^{-1 / 2}$ near the escape region. The expression (28) is confirmed on the exactly solvable cases presented in Appendices $\mathrm{A}$ and C. We also found a perfect agreement of Eq. (28) with two numerical simulations in which the target is (i) a square concentric to the confining disk; and (ii) a sphere included within the boundary of a sphere, both two and three dimensions (results are not shown).

Comparing approximate relations (27 28), we define the transition death rate $\mu_{c}$ between two asymptotic regimes:

$$
\mu_{c}=\frac{(|\Omega| /|\Gamma|)^{2}}{D{\overline{\left\langle\tau_{0}\right\rangle}}^{2}} .
$$

Substituting the asymptotic relation (18) for the global MFPT, one finds that $\mu_{c} \sim D / a^{2}$ in both two and three dimensions, where $a$ is the size of the escape region.

\section{First passage position}

While we mainly focused on the FPT to the escape region, the identification and further isolation of the most probable exit locations is also of practical importance. In this section, we show how the distribution of first passage positions (FPP) can be expressed through the MFPT of mortal walkers.

For this purpose, we recall that the probability flux density,

$$
q\left(t, x ; x_{0}\right)=-D \frac{\partial}{\partial n} G_{t}\left(x ; x_{0}\right),
$$

obtained from the diffusion propagator $G_{t}\left(x ; x_{0}\right)$, can be interpreted as the joint probability density for the FPT and the FPP on the escape region (e.g., see 29]). In particular, the integral of this function over $x \in \Gamma$ yields the marginal probability density $\rho\left(t ; x_{0}\right)$ for the FPT,

$$
\rho\left(t ; x_{0}\right)=\int_{\Gamma} d x q\left(t, x ; x_{0}\right)
$$

(see Appendix E). In turn, the integral over time $t$ yields the marginal probability density $\omega_{\mu}\left(x ; x_{0}\right)$ for the FPP of mortal walkers,

$$
\omega_{\mu}\left(x ; x_{0}\right)=\int_{0}^{\infty} d t q\left(t, x ; x_{0}\right) e^{-\mu t},
$$

where we explicitly included the factor $e^{-\mu t}$ to account for the first-order bulk reaction (see Appendix E for derivation). Finally, if the starting point $x_{0}$ is distributed

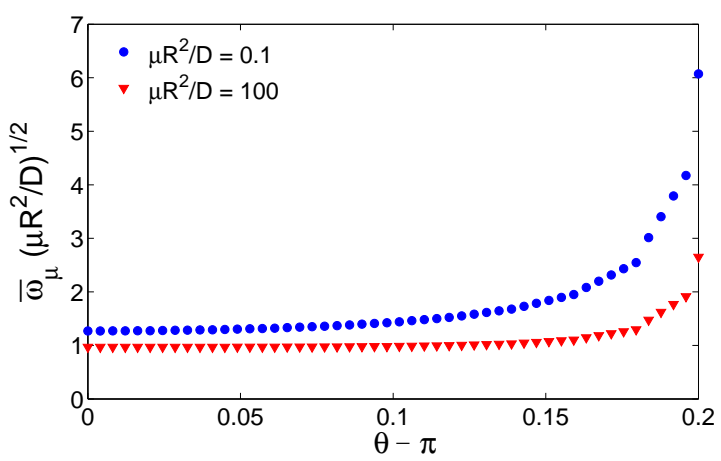

FIG. 4: (Color online) FPP probability density $\bar{\omega}_{\mu}(x)$ (given by Eq. (C14) ), multiplied by $\sqrt{\mu R^{2} / D}$, as a function of angular coordinate $\theta$ of the passage position $x$ on $\Gamma$, for the disk of radius $R$ with an escape arc $(\pi-\varepsilon, \pi+\varepsilon)$ and $\varepsilon=0.2$ (see Fig. 10). For a small death rate $\mu R^{2} / D=0.1$, the density greatly increases on the edges of the escape region (at $\theta=\varepsilon)$. For a larger death rate $\mu R^{2} / D=100$, the density becomes flatter, illustrating the convergence to a uniform distribution as $\mu \rightarrow \infty$. Note that only the half of the density, for $\pi \leq \theta \leq \pi+\varepsilon$, is shown (the other half being symmetric).

uniformly, one gets for any $x \in \partial \Omega$

$$
\begin{aligned}
\bar{\omega}_{\mu}(x) & =\frac{1}{|\Omega|} \int_{\Omega} d x_{0} \omega_{\mu}\left(x ; x_{0}\right)=-\frac{D}{|\Omega|} \int_{0}^{\infty} d t \frac{\partial}{\partial n} S(t ; x) e^{-\mu t} \\
& =-\frac{D}{|\Omega|} \frac{\partial \tilde{S}(\mu ; x)}{\partial n}=-\frac{D}{|\Omega|} \frac{\partial}{\partial n}\left\langle\tau_{\mu}\right\rangle=\frac{D}{\mu|\Omega|} \frac{\partial H_{\mu}(x)}{\partial n} .
\end{aligned}
$$

In other words, the MFPT for mortal walkers also determines their escape positions.

We illustrate the result (33) in the case of an escape from a ball of radius $R$, for which the MFPT $\left\langle\tau_{\mu}\right\rangle$ is given in Eq. (A1). In the limit of small death rates, the distribution converges to $\bar{\omega}_{\mu} \sim 1 /|\Gamma|$, where $|\Gamma|=|\partial \Omega|$ is the surface of the $(d-1)$-sphere, which corresponds to the expected uniform measure over the sphere. At large death rates, we find that $\bar{\omega}_{\mu} \sim \frac{1}{|\Omega|}(\mu / D)^{-1 / 2}$ in any dimensions. The latter relation is expected since it corresponds to the product of the uniform measure on the sphere and the GEP defined in Eq. (28).

The expression (33) is of particular interest in the study of the narrow escape problem. As discussed in 29] for immortal walkers, the probability density $\bar{\omega}_{0}(\theta)$ diverges at the boundaries of the escape region. In other words, immortal walkers tend to exit through the edges of the escape region. We show that this proclivity is hindered at large death rates $\mu$, at which $\bar{\omega}_{\mu}(\theta) \sim(\mu / D)^{-1 / 2}$ (see Fig. 41). In the next section, we discuss the implications of the asymptotic behavior (33) at low and high death rates for the leakage control. 


\section{DISCUSSION}

\section{A. Leakage control}

In many real-life applications, one needs to fabricate efficient containers for a safe storage of dangerous species such as nuclear wastes or toxic chemicals. For this purpose, one can either improve the isolation of the container, or incorporate mechanisms to bind, transform, deactivate or disintegrate dangerous species in the bulk or on the boundary. In mathematical terms, the first strategy aims at shrinking escape regions to increase the $\operatorname{MFPT}\left\langle\tau_{0}\right\rangle$ for immortal walkers according to the asymptotic relation (18). Since a complete isolation is not realistic (e.g., due to a slow but permanent degradation of container materials), the exit probability of intact diffusing species (with $\mu=0$ ) would be equal to 1 . In other words, whatever the isolation improvements are, the leakage is only a matter of time. It is therefore important to implement in parallel the second strategy that aims at reducing the mean lifetime $1 / \mu$. Note that this is a natural frame for nuclear wastes which disintegrate by a radioactive decay.

The asymptotic analysis in Sec. III has shown how the $\operatorname{MFPT}\left\langle\tau_{\mu}\right\rangle$ and the exit probability $H_{\mu}\left(x_{0}\right)$ are affected by the finite lifetime of the walkers. When the mean lifetime $1 / \mu$ is much larger than the time $\left\langle\tau_{0}\right\rangle$ needed to find the escape region by immortal walkers, the decay mechanism weakly affects the escape process. In particular, the exit probability remains close to 1 , indicating on a poor leakage protection. Improving either the isolation, or the decay mechanism, or both, one aims at switching to the opposite limit $\mu\left\langle\tau_{0}\right\rangle \gg 1$, in which the exit probability can be significantly reduced.

In this regime, the MFPT $\left\langle\tau_{\mu}\right\rangle$ was shown to decrease universally as $1 / \mu$, i.e., this quantity essentially reflects the mean lifetime. In turn, the exit probability $H_{\mu}\left(x_{0}\right)$ remains informative and was shown to decay as stretchedexponential, $\exp \left(-\left|x_{0}-\Gamma\right| \sqrt{\mu / D}\right)$, when the death rate $\mu$ increases. While we could not provide a general form of the dependence of the exit probability $H_{\mu}\left(x_{0}\right)$ on the size of the escape region, we derived the asymptotic behavior of the exit probability for several specific domains (see Appendices $\mathrm{A}$ and $\mathrm{C}$ ).

The leakage of many species uniformly distributed in a container can be characterized by the global exit probability $\bar{H}_{\mu}$ which exhibits a much slower decay with $\mu$, as suggested by approximations (27 28). The approximation (27) can be interpreted as the result of competition of two first-order kinetics:

$$
\text { Dead } \underset{\mu}{\longleftarrow} \text { Alive } \underset{k_{e}}{\longrightarrow} \text { Exited }
$$

where $k_{e}=1 / \overline{\left\langle\tau_{0}\right\rangle}$ is the exit rate in absence of a "death mechanism". In the stationary state, the law of mass action 35] predicts that the proportion of dead $n_{D}$ to exited $n_{E}=1-n_{D}$ walkers reads $n_{D} / n_{E}=\mu \overline{\left\langle\tau_{0}\right\rangle}$, from which $n_{E}=1 /\left(1+\mu \overline{\left\langle\tau_{0}\right\rangle}\right)$. Since the proportion $n_{E}$ of exited walkers is precisely the exit probability $\bar{H}_{\mu}$, one recovers the approximation (27). One can conclude that the GEP remains close to 1 at small $\mu$ even for high quality isolation (small $\varepsilon$ ), whereas shrinking the escape region allows one to significantly improve the safety of a container when $\mu R^{2} / D \gtrsim 1$. At very large death rates, the above kinetic argument fails, and the GEP is determined by the universal asymptotic relation (28).

Note also that active protecting mechanisms can be implemented not only in the bulk, but also on the container boundary, including the escape regions. As shown in [36], the introduction of an energetic or entropic barrier at the escape region significantly affects the MFPT $\left\langle\tau_{0}\right\rangle$ for immortal walkers, making the escape process "barrierlimited" instead of "diffusion-limited". In particular, the conventional asymptotic behavior (18) is replaced by a faster divergence as $(\kappa / D) \epsilon^{1-d}$, where $\kappa$ is the barrier reactivity or permeability. In other words, the presence of energetic or entropic barrier greatly improves the quality of isolation and allows one to reduce the exit probability. In the same vein, long-ranged repulsive interactions keep diffusing species expelled from the boundary and thus increase the MFPT of immortal walkers 36. In addition, our general result (33) concerning the distribution of exit positions can be used to design protecting shields that would be adapted to the death rate of the toxic reactant. In particular, we showed that highly mortal walkers hit uniformly the escape region, in contrast to immortal walkers that mainly exit through the edges of the escape region (see discussion in Sec. IIIC).

\section{B. Scaling argument for mRNA translation}

As another application of our results, we propose a scaling argument to express the variability of the mRNA lifetime between E. Coli, Yeast and Human cells in terms of the cell volume and the number of ribosomes. In this biological context, the escape region is the surface of ribosomes, while the exit probability can be interpreted as the probability for an mRNA to encounter a ribosome before being degradated. Assuming that the asymptotic relation (28) holds (i.e., that mRNA translation occurs in the high degradation rate regime), we get the scaling relation

$$
\frac{N s_{r}}{V} \sqrt{D / \mu} \sim \bar{H}_{\mu}
$$

between the degradation rate $\mu$, the cell volume $V$, and the total ribosome surface $S=N s_{r}$ that mRNA should hit in order to trigger a translation, where $s_{r}$ is the typical reactive surface of one ribosome and $N$ is the number of ribosomes in the cell.

In addition, we assume that (i) the diffusion coefficient $D$ is the same for different species [37] and (ii) that an identical "success rate" $\bar{H}_{\mu}$ in the reaction kinetics should be maintained for all cells to ensure an efficient translation mechanism. From Eq. (35), the ratio $N /(V \sqrt{\mu})$ 


\begin{tabular}{|c|c|c|c|}
\hline & E. Coli & Yeast & Human \\
\hline$N$ & $10^{4}$ & $10^{5}$ & $10^{6}$ \\
$V\left(\right.$ in $\left.\mu \mathrm{m}^{3}\right)$ & 2 & 40 & 2000 \\
$1 / \mu($ in min $)$ & 5 & 20 & 600 \\
\hline $10^{-4} N /(V \sqrt{\mu})$ & 1.1 & 1.1 & 1.2 \\
$\left(\right.$ in $\left.\mu \mathrm{m}^{-3} \cdot \min ^{1 / 2}\right)$ & & & \\
\hline
\end{tabular}

TABLE I: The number of ribosomes $(N)$, the cell volume $(V)$, and the mRNA degradation rate $(\mu)$ for E. Coli, Yeast, and Human fibroblast cells [37].

should then be approximatively constant among different species. This scaling argument is confirmed by comparing E. Coli, Yeast, and Human cells (Table I). Using the known data for one cell type, one should thus be able to estimate the degradation rate of mRNA in another cell type from its volume and the number of ribosomes. Similar arguments could hold for the transcription problem, involving the search of a specific DNA site by transcription factors. As discussed in 32, it appears that only the fastest $0.01 \%$ to $1 \%$ of transcription factors actually matter for the cellular response.

In summary, our results for the GEP provides a new interpretation for the cell size scaling problem, which has received a large attention within the recent years (see [38] and references therein). By narrowing the spread of the first passage times, the degradation rate might be used to focus the temporal cellular response to external perturbations.

\section{Extensions}

The escape problem for mortal walkers can be extended in various ways. First, the diffusion equation in Eqs. (11) can be replaced by a more general backward Fokker-Planck (or Kolmogorov) equation to account for the effect of external forces or potentials [28]. For instance, external forces can model the effect of chemotactic gradient which was proved to be relevant for the egg search problem by spermatozoa [20]. Second, the Dirichlet boundary condition on the escape region can be replaced by a Robin condition to model the presence of a recognition step, partial reflections, stochastic gating, or microscopically heterogeneous distribution of exit channels [39-46]. The survival probability and the distribution of FPTs to the whole partially absorbing boundary have been earlier studied for immortal walkers [47 49]. Third, one can consider intermittent processes, with alternating phases of bulk and surface diffusion [50 55]. Fourth, one can investigate the effect of heterogeneous materials or multiple layers of a container with different diffusion or trapping properties (this is a typical situation for nuclear waste storage). Fifth, the retarding effect of geometric or energetic traps can be included by considering the continuous time random walk (CTRW) with a fat-tailed waiting time distribution [47, 48, 56, 59]. The extension of these problems to mortal walkers consists in replacing the Laplace transform variable $p$ by $p+\mu$, as in Eq. (7) for normal diffusion.

To illustrate this point, we consider an extension to CTRW for which the propagator obeys the fractional diffusion equation, while the Laplace-transformed survival probability satisfies the Helmholtz equation

$$
\left[p^{1-\alpha} D_{\alpha} \Delta-p\right] \tilde{S}^{\alpha}\left(p ; x_{0}\right)=-1
$$

with the same boundary conditions as for normal diffusion, where $0<\alpha<1$ is the scaling exponent, and $D_{\alpha}$ is the generalized diffusion coefficient (in units $\mathrm{m}^{2} / \mathrm{s}^{\alpha}$ ). Changing the variable $p$ to $D p^{\alpha} / D_{\alpha}$, the solution of this equation can be expressed through the earlier obtained $\tilde{S}\left(p ; x_{0}\right)$ :

$$
\tilde{S}^{\alpha}\left(p ; x_{0}\right)=\frac{p^{\alpha-1} D}{D_{\alpha}} \tilde{S}\left(D p^{\alpha} / D_{\alpha} ; x_{0}\right) .
$$

From this relation, one immediately retrieves that the MFPT of immortal walkers is infinite: $\left\langle\tau_{0, \alpha}\right\rangle=$ $\tilde{S}^{\alpha}\left(0 ; x_{0}\right)=\infty$ because the walkers can be trapped for long periods of time until they reach the escape region. In contrast, the MFPT for mortal walkers is finite:

$$
\left\langle\tau_{\mu, \alpha}\right\rangle=\frac{\mu^{\alpha-1} D}{D_{\alpha}} \tilde{S}\left(D \mu^{\alpha} / D_{\alpha} ; x_{0}\right)=\frac{\mu^{\alpha-1} D}{D_{\alpha}}\left\langle\tau_{D \mu^{\alpha} / D_{\alpha}}\right\rangle .
$$

In fact, too long trajectories whose contribution led to divergence of the MFPT for CTRW, are eliminated because of a finite lifetime of the walker. In other words, the MFPT for mortal CTRW is related to the MFPT for mortal normal walkers with a modified death rate: $\mu_{\alpha}=D \mu^{\alpha} / D_{\alpha}$. Finally, the exit probability for mortal CTRWs that can be defined in analogy with Eq. (10) as

$$
H_{\mu, \alpha}\left(x_{0}\right)=\int_{0}^{\infty} d t e^{-\mu t}\left(-\frac{\partial S^{\alpha}\left(t ; x_{0}\right)}{\partial t}\right),
$$

is simply

$$
H_{\mu, \alpha}\left(x_{0}\right)=H_{\mu_{\alpha}}\left(x_{0}\right) .
$$

One can therefore apply the results from Sec. IIIto mortal CTRWs.

In all these extensions, the first-order bulk kinetics or, equivalently, an exponentially distributed lifetime of the walker, controls the duration of trajectories, assigning smaller weights to longer trajectories. We also mention the possibility of considering other lifetime distributions $Q(t)$ beyond the exponential one. In this case, Eq. (6) is still applicable but one needs to get the survival probability in time domain by inverse Laplace transform. Finally, if the death mechanism is coupled to diffusion (e.g., in the case of a space-dependent death rate), one has to treat the whole diffusion-reaction mixed boundary value problem. 


\section{CONCLUSION}

We formulated the escape problem to mortal walkers and investigated how their finite lifetime drastically affects the survival and exit probabilities. The latter is the likelihood of escape or leakage from the confining container and can thus characterize its isolation quality. We focused on the most relevant case of a first-order bulk kinetics or, equivalently, an exponentially distributed lifetime, for which the problem is reduced to finding the Laplace-transformed survival probability for immortal walkers. We derived the upper and lower bounds for the $\operatorname{MFPT}\left\langle\tau_{\mu}\right\rangle$ and the exit probability $H_{\mu}\left(x_{0}\right)$ and analyzed their asymptotic behavior at small and large death (or reaction) rates. When the mean lifetime, $1 / \mu$, is much larger than the MFPT for immortal walkers, $\left\langle\tau_{0}\right\rangle$, the exit probability remains close to 1 , meaning a poor isolation. In this situation, the leakage of dangerous species is just a matter of time. For a safer protection, one needs both to improve the boundary isolation by shrinking escape regions (thus increasing $\left\langle\tau_{0}\right\rangle$ ), and to implement efficient trapping, binding or deactivation mechanisms (thus increasing $\mu$ ). Improving only one of these aspects is not sufficient to significantly reduce $H_{\mu}\left(x_{0}\right)$. For the volumeaveraged, or global exit probability $\bar{H}_{\mu}$ that quantifies the overall safety of a container, we obtained two approximations at intermediate and large death rates. The quality of the obtained analytical results for general confining domains was confirmed by comparison with several explicitly solvable cases, and with numerical simulations. We also introduced and investigated the distribution of the first passage positions as a mathematical ground for design and optimization of containers. The density $\bar{\omega}_{\mu}(x)$ was shown to exhibit a transition from a singular function highly localized near edges of the escape region for immortal walkers, to asymptotically uniform density at large death rates. Various extensions and applications of the studied escape problem have been discussed including the leakage control of dangerous chemicals and scaling relation for mRNA translation mechanism.

\section{Acknowledgments}

The authors acknowledge the support under Grant No. ANR-13-JSV5-0006-01 of the French National Research Agency. We thank T. Saunders and M. Howard for interesting discussions about the cell size regulation problem.

\section{Appendix A: Explicit results for rotation-invariant domains}

\section{Escape from a ball}

The escape through the whole boundary of a ball is the simplest and the most studied case. The separation of variable allows one to derive an explicit spectral representation of the propagator in terms of Laplacian eigenfunctions, from which other quantities are deduced [1, 28]. In particular, the Laplace-transformed survival probability from the ball $B_{d}(R)$ of radius $R$ in $\mathbb{R}^{d}$ reads (e.g., see [48, 56]):

$$
\tilde{S}_{B_{d}(R)}\left(p ; x_{0}\right)=\frac{1}{p}\left(1-\left(\frac{\left|x_{0}\right|}{R}\right)^{1-\frac{d}{2}} \frac{I_{\frac{d}{2}-1}\left(\left|x_{0}\right| \sqrt{p / D}\right)}{I_{\frac{d}{2}-1}(R \sqrt{p / D})}\right),
$$

where $I_{\nu}(z)$ is the modified Bessel function of the first kind, and $x_{0}$ is the starting point. From this expression, one can immediately deduce the MFPT, the exit probability and the GEP for mortal walkers. The asymptotic behavior at small and large $p$ can be easily obtained.

In one dimension, this is the escape problem from an interval $(-R, R)$ with two escape points $\pm R$ or, equivalently, from an interval $(0, R)$ with one escape point at $x=R$ and reflecting endpoint at $x=0$. In this case, Eq. (A1) reduces to

$$
\left\langle\tau_{\mu}\right\rangle=\frac{1}{\mu}\left(1-\frac{\cosh \left(x_{0} \sqrt{\mu / D}\right)}{\cosh (R \sqrt{\mu / D})}\right)
$$

from which the exit probability and the GEP read as

$$
H_{\mu}\left(x_{0}\right)=\frac{\cosh \left(x_{0} \sqrt{\mu / D}\right)}{\cosh (R \sqrt{\mu / D})}
$$

and

$$
\bar{H}_{\mu}=\frac{\tanh (R \sqrt{\mu / D})}{R \sqrt{\mu / D}} \underset{\mu \rightarrow \infty}{\sim} \frac{1}{R \sqrt{\mu / D}} .
$$

In two dimensions, one gets the MFPT and global MFPT

$$
\left\langle\tau_{\mu}\right\rangle=\frac{1}{\mu}\left(1-\frac{I_{0}(r \sqrt{\mu / D})}{I_{0}(R \sqrt{\mu / D})}\right)
$$

and

$$
\overline{\left\langle\tau_{\mu}\right\rangle}=\frac{1}{\mu}\left(1-\frac{2 I_{1}(R \sqrt{\mu / D})}{R \sqrt{\mu / D} I_{0}(R \sqrt{\mu / D})}\right),
$$

where $r=\left|x_{0}\right|$. The exit probability and the GEP read

$$
H_{\mu}(r)=\frac{I_{0}(r \sqrt{\mu / D})}{I_{0}(R \sqrt{\mu / D})} \underset{\mu \rightarrow \infty}{\sim} \frac{e^{-(R-r) \sqrt{\mu / D}}}{\sqrt{r / R}}
$$

and

$$
\bar{H}_{\mu}=\frac{2 I_{1}(R \sqrt{\mu / D})}{R \sqrt{\mu / D} I_{0}(R \sqrt{\mu / D})} \underset{\mu \rightarrow \infty}{\sim} \frac{2}{R \sqrt{\mu / D}} .
$$

This asymptotic behavior agrees with Eq. (28). 
In three dimensions, the expressions for the MFPT and global MFPT are

$$
\left\langle\tau_{\mu}\right\rangle=\frac{R e^{(R-r) \sqrt{\mu / D}}-R e^{(R+r) \sqrt{\mu / D}}+r e^{2 R \sqrt{\mu / D}}-r}{\mu r\left(e^{2 R \sqrt{\mu / D}}-1\right)}
$$

and

$$
\overline{\left\langle\tau_{\mu}\right\rangle}=\frac{1}{\mu}\left(1-\frac{3 R \sqrt{\mu / D} \operatorname{coth}(R \sqrt{\mu / D})+3}{R^{2} \mu / D}\right) .
$$

The exit probability reads

$$
H_{\mu}(r)=\frac{R \sinh (r \sqrt{\mu / D})}{r \sinh (R \sqrt{\mu / D})} \underset{\mu \rightarrow \infty}{\sim} \frac{R}{r} e^{-(R-r) \sqrt{\mu / D}},
$$

where the limit is taken for starting points $x_{0}$ sufficiently far away from the boundary. The GEP reads

$$
\bar{H}_{\mu}=3 \frac{R \sqrt{\mu / D} \operatorname{coth}(R \sqrt{\mu / D})-1}{R^{2} \mu / D} \underset{\mu \rightarrow \infty}{\sim} \frac{3}{R \sqrt{\mu / D}},
$$

in agreement with Eq. (28).

\section{Concentric escape region}

For most applications, however, the escape region presents only a small part of the boundary. We consider another example of rotation-invariant domains with two concentric boundaries, $\left\{x \in \mathbb{R}^{d}: a<|x|<R\right\}$, with the escape region at the inner boundary of radius $a$, while the outer boundary of radius $R$ is fully reflecting. The rotation invariance of the domain leads to explicit representations of the Laplace-transformed survival probability, the exit probability and the GEP. In particular, Eq. (3) becomes

$$
\left(\frac{\partial^{2}}{\partial r^{2}}+\frac{d-1}{r} \frac{\partial}{\partial r}-\frac{p}{D}\right) \tilde{S}(p ; r)=-\frac{1}{D}
$$

subject to two boundary conditions:

$$
\tilde{S}(p ; a)=0, \quad\left(\frac{\partial}{\partial r} \tilde{S}(p ; r)\right)_{r=R}=0 .
$$

In two dimensions, the solution of this equation is

$$
\tilde{S}(p ; r)=\frac{1}{p}\left[1-\frac{I_{1}(\sqrt{s}) K_{0}\left(\sqrt{s} \frac{r}{R}\right)+K_{1}(\sqrt{s}) I_{0}\left(\sqrt{s} \frac{r}{R}\right)}{I_{1}(\sqrt{s}) K_{0}\left(\sqrt{s} \frac{a}{R}\right)+K_{1}(\sqrt{s}) I_{0}\left(\sqrt{s} \frac{a}{R}\right)}\right],
$$

where $s=p R^{2} / D$ and $K_{\nu}(z)$ is the modified Bessel function of the second kind. From this expression, one gets

$$
H_{\mu}(r)=\frac{I_{1}(\sqrt{s}) K_{0}\left(\sqrt{s} \frac{r}{R}\right)+K_{1}(\sqrt{s}) I_{0}\left(\sqrt{s} \frac{r}{R}\right)}{I_{1}(\sqrt{s}) K_{0}\left(\sqrt{s} \frac{a}{R}\right)+K_{1}(\sqrt{s}) I_{0}\left(\sqrt{s} \frac{a}{R}\right)},
$$

with $s=\mu R^{2} / D$, while the GEP reads

$$
\bar{H}_{\mu}=\frac{2 \frac{a}{R} s^{-1 / 2}\left[I_{1}(\sqrt{s}) K_{1}\left(\sqrt{s} \frac{a}{R}\right)-K_{1}(\sqrt{s}) I_{1}\left(\sqrt{s} \frac{a}{R}\right)\right]}{\left(1-\left(\frac{a}{R}\right)^{2}\right)\left[I_{1}(\sqrt{s}) K_{0}\left(\sqrt{s} \frac{a}{R}\right)+K_{1}(\sqrt{s}) I_{0}\left(\sqrt{s} \frac{a}{R}\right)\right]} .
$$

Note that the MFPT for immortal walkers is

$$
\left\langle\tau_{0}\right\rangle=\tilde{S}(0 ; r)=\frac{R^{2} \ln (r / a)}{2 D}-\frac{r^{2}-a^{2}}{4 D},
$$

that diverges as $\ln (1 / a)$ for $a \rightarrow 0$, as expected.

In three dimensions, the solution of Eq. A13 is

$$
\tilde{S}(p ; r)=\frac{1}{p}\left[1-\frac{a}{r} e^{-\sqrt{s}(r-a) / R} \frac{\frac{\sqrt{s}-1}{\sqrt{s}+1}+e^{-2 \sqrt{s}(1-r / R)}}{\frac{\sqrt{s}-1}{\sqrt{s}+1}+e^{-2 \sqrt{s}(1-a / R)}}\right],
$$

from which

$$
H_{\mu}(r)=\frac{a}{r} e^{-\sqrt{s}(r-a) / R} \frac{\frac{\sqrt{s}-1}{\sqrt{s}+1}+e^{-2 \sqrt{s}(1-r / R)}}{\frac{\sqrt{s}-1}{\sqrt{s}+1}+e^{-2 \sqrt{s}(1-a / R)}},
$$

and

$\bar{H}_{\mu}=\frac{3 \frac{a}{R}\left(\frac{\sqrt{s}-1}{\sqrt{s}+1}\left(1+\frac{a}{R} \sqrt{s}\right)+e^{-2 \sqrt{s}(1-a / R)}\left(1-\frac{a}{R} \sqrt{s}\right)\right)}{s\left(1-\frac{a^{3}}{R^{3}}\right)\left(\frac{\sqrt{s}-1}{\sqrt{s}+1}+e^{-2 \sqrt{s}(1-a / R)}\right)}$.

Note that the MFPT for immortal walkers is

$$
\left\langle\tau_{0}\right\rangle=\tilde{S}(0 ; r)=\frac{R^{3}(r-a)}{3 D r a}-\frac{r^{2}-a^{2}}{6 D},
$$

that diverges as $1 / a$ for $a \rightarrow 0$, as expected.

The explicit form of the exit probability, $H_{\mu}\left(x_{0}\right)$, and the GEP, $\bar{H}_{\mu}$, helps us to investigate three asymptotic regimes of weak $\left(\mu \ll D / R^{2}\right)$, intermediate $\left(D / R^{2} \ll\right.$ $\left.\mu \ll D / a^{2}\right)$, and strong $\left(\mu \gg D / a^{2}\right)$ death rates. Note that the second regime emerges only under the narrow escape condition $a \ll R$ that we assume here. We start with the three-dimensional case for which the analysis is much simpler.

In the weak death rate regime, the Taylor expansion (16) reduces the analysis to computing the moment of $\tau_{0}$ which are given by Eq. (17) and can be found explicitly from the exact formula (A20). When $\mu \gg D / R^{2}$, the GEP from Eq. A21 is greatly simplified to

$$
\bar{H}_{\mu} \simeq \frac{3 a(1+a \sqrt{\mu / D})}{R^{3} \mu / D},
$$

from which the intermediate and strong death rate regimes can be distinguished:

$$
\bar{H}_{\mu} \simeq \frac{3 a}{R^{3}} \times\left\{\begin{array}{lr}
D / \mu & \left(D / R^{2} \ll \mu \ll D / a^{2}\right), \\
a \sqrt{D / \mu} & \left(\mu \gg D / a^{2}\right) .
\end{array}\right.
$$


The latter asymptotic regime agrees with Eq. (28). In both regimes, the exit probability is simplified when $r$ is not too close to $R$

$$
H_{\mu}(r) \simeq \frac{a}{r} \exp (-(r-a) \sqrt{\mu / D}) .
$$

In two dimensions, one gets

$$
\bar{H}_{\mu} \sim \frac{2}{R^{2} \mu / D\left(-\gamma-\ln \frac{a \sqrt{\mu / D}}{2}\right)}
$$

for intermediate death rates, and

$$
\bar{H}_{\mu} \underset{\mu \rightarrow \infty}{\sim} \frac{2 a}{R^{2} \sqrt{\mu / D}}
$$

in the limit of strong death rates $\left(\mu \gg D / a^{2}\right)$, where $\gamma$ is the Euler constant. Again, this result agrees with the general relation (28). In the limit $\mu \gg D / R^{2}$ (or $s \gg 1$ ), one gets

$$
H_{\mu}(r) \underset{\mu \rightarrow \infty}{\sim} \frac{\sqrt{\pi} e^{-r \sqrt{\mu / D}}\left(1+e^{-2(R-r) \sqrt{\mu / D}}\right)}{\sqrt{2}\left(r^{2} \mu / D\right)^{1 / 4}\left(\gamma+\ln \frac{a \sqrt{\mu / D}}{2}\right)} .
$$

\section{Appendix B: Exit time from a ball with reflecting obstacles}

The lower bound of the Laplace-transformed survival probability relies on the inequality (21) which states that the FPT from a point $x_{0}$ to the escape region $\Gamma$ is greater than the FPT from the origin of the ball $B=B_{d}\left(\left|x_{0}-\Gamma\right|\right)$ of radius $\left|x_{0}-\Gamma\right|$ to its boundary. This property is based on two statements: (i) the FPT to the escape region is greater than the first exit time from the subdomain $\Omega \cap B$ through the boundary of the ball $B$, and (ii) the latter is equal to or greater than the FPT from the origin of the ball $B$ to its boundary. The first statement follows from the continuity of Brownian motion: before reaching $\Gamma$, a particle must cross the boundary of the ball $B$. However, the second statement is less evident. It claims that the presence of reflecting obstacles inside a ball (here, the boundary $\partial \Omega \cap B$ ) cannot speed up the exit from this ball (Fig. 5a). Qualitatively, this statement sounds natural: reflecting obstacles "shield" some boundary points from the center and thus potentially increase the FPT. Note also that the presence of reflecting obstacles may not affect the FPT at all, as illustrated by the case of a sector, for which the FPT is exactly the same as for the whole disk (Fig. 5b).

We mention that this claim cannot be generalized to any starting point or any domain. Indeed, if the starting point was not at the origin of the ball, adding a concentric spherical reflecting obstacle of radius $r=\left|x_{0}\right|$ would certainly speed up the exit. For instance, for an annulus $r<|x|<R$ with reflecting inner circle at $r$, the MFPT

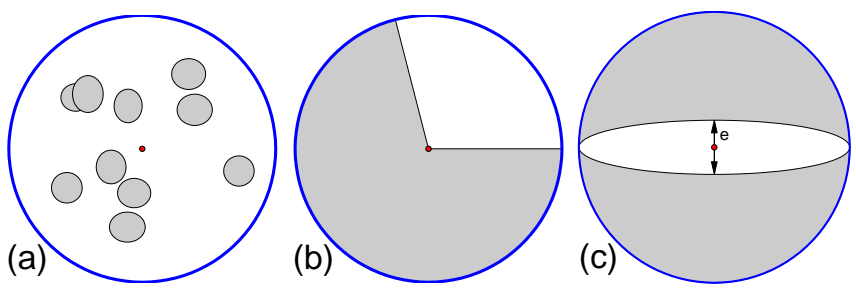

FIG. 5: (Color online) Illustrations of the escape problem from a ball with reflecting obstacles (gray shadowed regions).

from the starting point $(r, \theta)$ (in polar coordinates) to the outer circle at $R$ is

$$
\left\langle\tau_{0}\right\rangle=\frac{R^{2}-r^{2}}{4 D}+\frac{r^{2}}{2 D} \ln (r / R) .
$$

The first term, which is the MFPT for the disk without obstacles, is decreased by the second negative term.

Without having a rigorous mathematical proof for this statement, we illustrate it on several examples. First, let us consider a case when the reflecting obstacles covers most of the volume of the ball $B_{d}(R)$ (see Fig. 5r). When the width $e$ of the remaining domain accessible to diffusion shrinks to zero, the search process becomes $(d-1)$-dimensional (e.g., for $d=2$, the walkers diffuse along one-dimensional interval $(-R, R)$ and search for its endpoints). We expect that the survival probability at any given time $t$ increases as $e$ decreases. This behavior is exemplified by considering the limiting case $e=0$ : the survival probability $S_{B_{d-1}(R)}(t ; 0)$ (i.e., when the escape region $\Gamma$ is the boundary of $(d-1)$ dimensional ball $\left.B_{d-1}(R)\right)$ is larger than the survival probability $S_{B_{d}(R)}(t ; 0)$ that corresponds to the escape from the original ball $B_{d}(R)$. This follows from the fact that $\tilde{S}_{B_{d}(R)}(\mu ; 0)$ from Eq. (23) is a decreasing function of the dimensionality $d$ for all $\mu \geq 0$. In other words, the Laplace-transformed survival probability from the center of the ball $B_{d}(R)$ is smaller than its counterpart in the lower dimension $d-1$, i.e., $S_{B_{d-1}(R)}(\mu ; 0)$. One can see that drastic volume occupancy by reflecting obstacles increases the FPT. Note also that the MFPT from the center of a ball without obstacles, $R^{2} /(2 d D)$, also increases with the dimensionality reduction.

Second, we consider the disk $B_{2}(R)$ with regularly distributed reflecting circular obstacles, as illustrated in Fig. 6a,b. Solving the problem numerically, we obtain the Laplace-transformed survival probability in this configuration and compare it with $\tilde{S}_{B_{2}}(\mu ; 0)$ from Eq. (23). Figure 6r,d shows the relative difference between these two quantities, confirming again that the obstacles contribute to an increase of the survival probability. In spite of various qualitative and numerical evidences, our claim remains conjectural from a mathematical point of view. 

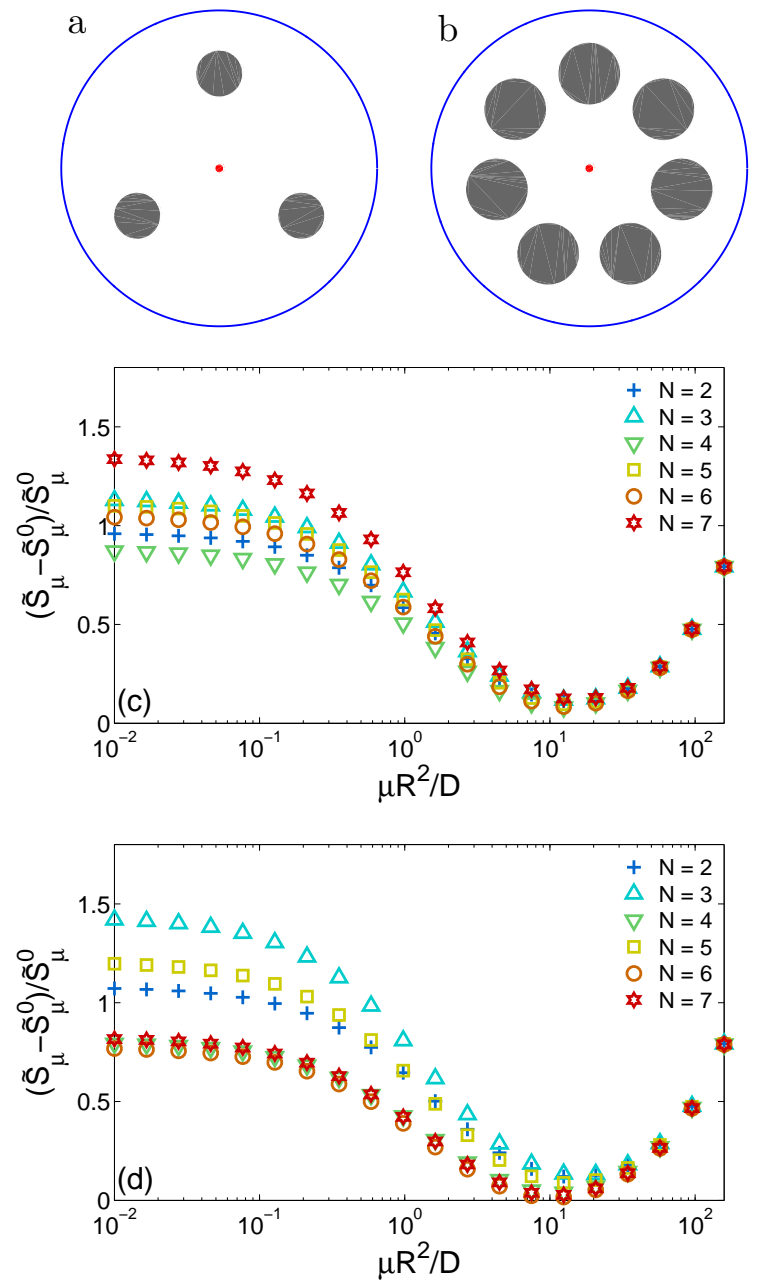

FIG. 6: (Color online) (a,b) Examples of regular arrangments of reflecting circular obstacles (gray shadowed regions) of radius $r$ inside the unit disk, with $r=0.1$ (a) and $r=0.2$ (b). (c-d) Relative difference between the Laplacetransformed survival probability in the presence of obstacles, $\tilde{S}_{\mu}$ (computed numerically), and that without obstacles, $\tilde{S}_{\mu}^{0}=\tilde{S}_{B_{d}(R)}(\mu ; 0)$ (given by Eq. (23)), as a function of the dimensionless death rate $\mu R^{2} / D$ for small obstacles $(r=0.1)$ (c) and larger obstacles $(r=0.2)(\mathbf{d})$. Note that the difference is always positive, indicating that reflecting obstacles inside a ball increase the exit time from the ball, in agreement with the inequality (21).

\section{Appendix C: Escape from the disk through an arc on the boundary}

The exact solution for the Laplace-transformed survival probability was obtained for immortal walkers in several planar domains such as a disk, an angular sector, an annulus, and a rectangle [30]. For the sake of completeness, we reproduce the exact and approximate solutions for the Laplace-transformed survival probability of a walker inside the disk of radius $R$ with the reflecting boundary except for an escape $\operatorname{arc} \Gamma=\{(R, \theta): \theta \in$ $(\pi-\varepsilon, \pi+\varepsilon)\}$ of angle $2 \varepsilon$. From these expressions, we easily get the MFPT and the exit probability for mortal walkers as discussed in the text. We also investigate their asymptotic behavior.

\section{Exact solution} [30]

The Laplace-transformed survival probability reads

$$
\tilde{S}\left(p ; r_{0}, \theta_{0}\right)=\tilde{S}^{\pi}\left(p ; r_{0}\right)+\frac{R^{2}}{D} u\left(p R^{2} / D ; r_{0} / R, \theta_{0}\right),
$$

where

$$
\tilde{S}^{\pi}\left(p ; r_{0}\right)=\frac{1}{p}\left(1-\frac{I_{0}\left(r_{0} \sqrt{p / D}\right)}{I_{0}(R \sqrt{p / D})}\right)
$$

is the rotation-invariant solution for the whole absorbing circle (see Eq. (A5)), while the auxiliary function $u$ (which is positive because $S\left(t ; x_{0}\right) \geq S_{\pi}\left(t ; x_{0}\right)$ ) can be expressed as

$$
u(s ; r, \theta)=\sum_{n=0}^{\infty} a_{n}(s) \frac{I_{n}(r \sqrt{s})}{I_{n}(\sqrt{s})} \cos (n \theta),
$$

where the coefficients $a_{n}(s)$ are uniquely determined through the boundary conditions (note that we replace the notation $a_{0}(s) / 2$ by $a_{0}(s)$ as compared to [30]):

$$
\begin{aligned}
& a_{0}(s)=C_{\varepsilon}(s)\left[\frac{\sqrt{s} I_{0}(\sqrt{s})}{I_{1}(\sqrt{s})}+s C_{\varepsilon}(s)\right]^{-1}, \\
& a_{n}(s)=2 \hat{a}_{n}(s)\left[\frac{\sqrt{s} I_{0}(\sqrt{s})}{I_{1}(\sqrt{s})}+s C_{\varepsilon}(s)\right]^{-1},
\end{aligned}
$$

where the auxiliary coefficients $\hat{a}_{n}(s)$ are determined as

$$
\hat{a}_{n}(s)=\left[(I-M \gamma)^{-1} \alpha\right]_{n} \quad(n \geq 1) .
$$

In this relation, $I$ is the identity matrix, $\gamma$ is the diagonal matrix formed by

$$
\gamma_{n}(s)=1-\frac{\sqrt{s}}{2 n} \frac{I_{n-1}(\sqrt{s})+I_{n+1}(\sqrt{s})}{I_{n}(\sqrt{s})} \quad(n \geq 1),
$$

$\alpha$ is the vector formed by

$$
\alpha_{n}=\frac{(-1)^{n-1}}{2 n}\left[P_{n-1}(\cos \varepsilon)+P_{n}(\cos \varepsilon)\right] \quad(n \geq 1)
$$

$\left(P_{n}(z)\right.$ being Legendre polynomials), the matrix $M$ is

$$
M_{n m}=\frac{m}{2} \int_{-\cos \varepsilon}^{1} \frac{d x}{1+x}\left[P_{m-1}(x)+P_{m}(x)\right]\left[P_{n-1}(x)+P_{n}(x)\right]
$$


(for $m, n \geq 1$ ), and

$$
C_{\varepsilon}(s)=-2 \ln \sin (\varepsilon / 2)+2 \sum_{n=1}^{\infty} n \alpha_{n} \hat{a}_{n}(s) \gamma_{n}(s) .
$$

From Eq. (C1), we deduce the exit probability $H_{\mu}\left(x_{0}\right)$ :

$$
\begin{aligned}
H_{\mu}\left(x_{0}\right) & =\left(\frac{\sqrt{s} I_{0}(\sqrt{s})}{I_{1}(\sqrt{s})}+s C_{\varepsilon}(s)\right)^{-1}\left[\frac{\sqrt{s} I_{0}\left(\frac{r_{0}}{R} \sqrt{s}\right)}{I_{1}(\sqrt{s})}\right. \\
& \left.-2 s \sum_{n=1}^{\infty} \hat{a}_{n}(s) \frac{I_{n}\left(\frac{r_{0}}{R} \sqrt{s}\right)}{I_{n}(\sqrt{s})} \cos n \theta_{0}\right],
\end{aligned}
$$

with $s=\mu R^{2} / D, r_{0}=\left|x_{0}\right|$, and $\theta_{0}$ being the angular coordinate of the starting point $x_{0}$. Setting $r_{0}=0$, one gets

$$
H_{\mu}(0)=\left(\frac{\sqrt{s} I_{0}(\sqrt{s})}{I_{1}(\sqrt{s})}+s C_{\varepsilon}(s)\right)^{-1} \frac{\sqrt{s}}{I_{1}(\sqrt{s})},
$$

from which Eq. Can be re-written as

$$
\begin{aligned}
H_{\mu}\left(x_{0}\right) & =H_{\mu}(0)\left[I_{0}\left(\frac{r_{0}}{R} \sqrt{s}\right)\right. \\
& \left.-2 \sqrt{s} I_{1}(\sqrt{s}) \sum_{n=1}^{\infty} \hat{a}_{n}(s) \frac{I_{n}\left(\frac{r_{0}}{R} \sqrt{s}\right)}{I_{n}(\sqrt{s})} \cos n \theta_{0}\right] .
\end{aligned}
$$

If the starting point $x_{0}$ is uniformly distributed over the disk, the volume average yields the GEP

$$
\begin{aligned}
\bar{H}_{\mu} & \equiv \frac{1}{\pi R^{2}} \int_{0}^{R} d r_{0} r_{0} \int_{0}^{2 \pi} d \theta_{0} H_{\mu}\left(r_{0}, \theta_{0}\right) \\
& =\frac{2 I_{1}(\sqrt{s})}{\sqrt{s}} H_{\mu}(0)=2\left(\frac{\sqrt{s} I_{0}(\sqrt{s})}{I_{1}(\sqrt{s})}+s C_{\varepsilon}(s)\right)^{-1} .
\end{aligned}
$$

Finally, the volume-averaged probability density of the FPP from Eq. (33) reads

$\bar{\omega}_{\mu}(\theta)=\frac{1}{\pi R}\left[\frac{I_{1}(\sqrt{s})}{\sqrt{s} I_{0}(\sqrt{s})}-\sqrt{s} \sum_{n=0}^{\infty} \hat{a}_{n}(s) \frac{I_{n}^{\prime}(\sqrt{s})}{I_{n}(\sqrt{s})} \cos n \theta_{0}\right]$.

Note that if the escape region is the whole boundary (i.e., $\varepsilon=\pi$ ), all $\alpha_{n}=0$ that implies $C_{\pi}(s)=0$ and $a_{n}(s)=\hat{a}_{n}(s)=0$ for $n \geq 1$. In particular, one gets

$$
\bar{H}_{\mu} \simeq \frac{2}{R \sqrt{\mu / D}} \quad\left(\mu \gg D / R^{2}\right)
$$

in agreement with the general asymptotic relation (28).

Moreover, if now the relation (28) is applied for a given size $\varepsilon$ of the escape region,

$$
\bar{H}_{\mu} \simeq \frac{2 \varepsilon / \pi}{R \sqrt{\mu / D}} \quad\left(\mu \gg D / R^{2}\right)
$$

Eq. (C13) implies the asymptotic behavior for $H_{\mu}(0)$

$$
H_{\mu}(0) \simeq \frac{\varepsilon \sqrt{2}}{\sqrt{\pi}}\left(R^{2} \mu / D\right)^{\frac{1}{4}} e^{-R \sqrt{\mu / D}} \quad\left(\mu \gg D / R^{2}\right) .
$$

Interestingly, this behavior is different from the case of the concentric escape region, for which Eq. (A28) yields for $r=R$ :

$$
H_{\mu}(R) \underset{\mu \rightarrow \infty}{\sim} \frac{\sqrt{2 \pi} e^{-R \sqrt{\mu / D}}}{\left(R^{2} \mu / D\right)^{\frac{1}{4}}\left(\gamma+\ln \frac{\varepsilon R \sqrt{\mu / D}}{2 \pi}\right)},
$$

where the radius $a$ of the escape region was expressed as $a=R \varepsilon / \pi$ so get the same perimeter of the escape region. The dependence of the exit probability on the size of the escape region is very different in both cases.

\section{Approximate solution for small $\varepsilon$}

The above solution given by Eqs. C1 - C13 is exact and valid for any size $2 \varepsilon$ of the escape region. However, this solution is not explicit as the inversion of the infinitedimensional matrix in Eq. C5 is needed. In practice, the matrices $M$ and $\gamma$ can be truncated to a finite size and then inverted numerically, leading to the solution with any desired accuracy.

For small $\varepsilon$, the matrix $M$ was shown to be close to the identity, yielding an explicit approximate solution,

$$
\begin{aligned}
\hat{a}_{n}(s) & \simeq \frac{\alpha_{n}}{1-\gamma_{n}(s)} \\
C(s) & \simeq-2 \ln \sin (\varepsilon / 2)+2 \sum_{n=1}^{\infty} \frac{n \alpha_{n}^{2} \gamma_{n}(s)}{1-\gamma_{n}(s)}
\end{aligned}
$$

that was shown to be very accurate for $\varepsilon$ small enough [30]. In particular, the approximate expression for $C(s)$ determines explicitly the global exit probability according to Eq. (C13).

Using these relations, one can investigate the asymptotic behavior of the survival probability, the MFPT, and the exit probability $H_{\mu}\left(x_{0}\right)$ in the narrow escape limit $(\varepsilon \ll 1)$. Here, we focus only on the global exit probability $\bar{H}_{\mu}$ as the most important safety characteristic of a container. For small $\mu$, one has $\gamma_{n}(s) \simeq-s /(2 n(n+1))$, from which

$$
C(s) \simeq 2 \ln (2 / \varepsilon)+O(s),
$$

and Eq. (C13) implies

$$
\bar{H}_{\mu} \simeq \frac{1}{1+\left(\mu R^{2} / D\right) \ln (2 / \varepsilon)}
$$

Given that $\left\langle\tau_{0}\right\rangle \simeq \frac{R^{2}}{D} \ln (2 / \varepsilon)$, this expression agrees with our general approximation (27) for intermediate death rates. 


\section{Quality of the upper bound (26)}

In this section, we examine the quality of the upper bound 26 in the case of a disk. Figure 7 a shows the exit probability $H_{\mu}(0)$ for the disk, normalized by the upper bound $U_{2}(R \sqrt{\mu / D})$, as a function of $\varepsilon$, for several values of the death rate $\mu$. One can see that, at large $\mu$, this ratio becomes proportional to $\varepsilon / \pi$. This result agrees with the following qualitative picture: at large $\mu$, the arrival onto the boundary is a rare event; only short trajectories that rapidly reach the boundary, can provide a notable contribution. If such a trajectory arrives onto the reflecting part of the boundary, it needs extra time to diffuse towards the escape region, and thus provides a much smaller contribution. As a consequence, the overall probability to reach the boundary, which is given by the upper bound, is multiplied by the fraction of trajectories arriving to the escape region, i.e., $\varepsilon / \pi$.

When the boundary points are not equally distant from the starting point, their contributions are not equal, and more complicated dependence on the escape region size is expected. This is illustrated in Fig. 7b showing the ratio $H_{\mu}\left(x_{0}\right) / H_{\mu}^{\mathrm{up}}\left(x_{0}\right)$ at the starting point $x_{0}=-0.5$ (i.e., $r_{0}=0.5$ and $\left.\theta_{0}=\pi\right)$ which is closer to the escape region. This ratio first grows proportionally to $\varepsilon$ (at small $\varepsilon$ ) but then saturates to a constant because any further addition of distant boundary points does not facilitate the escape from the disk at large $\mu$.

\section{Appendix D: Upper bound of the survival probability for the whole absorbing boundary}

The proposed upper bound (19) of the Laplacetransformed survival probability, which follows from a trivial inequality $S\left(t, x_{0}\right) \leq 1$, implies a useless lower bound for the exit probability: $H_{\mu}\left(x_{0}\right) \geq 0$. Finding more informative lower bounds for $H_{\mu}\left(x_{0}\right)$ is an interesting perspective. In this Appendix, we propose one improvement which, however, is only valid in the case when the escape region is the whole boundary: $\Gamma=\partial \Omega$.

The domain monotonicity for Dirichlet heat kernels 60] implies the general upper bound for the survival probability in a domain $\Omega \subset \mathbb{R}^{d}$ with fully reactive boundary $\partial \Omega$ :

$$
S\left(t ; x_{0}\right) \leq \int_{\Omega} d x \frac{\exp \left(-\frac{\left|x-x_{0}\right|^{2}}{4 D t}\right)}{(4 \pi D t)^{d / 2}}
$$

where the Gaussian propagator is explicitly written on the right-hand side. Splitting the integral into two contributions, from the ball of radius $\left|x_{0}-\partial \Omega\right|$ centered at
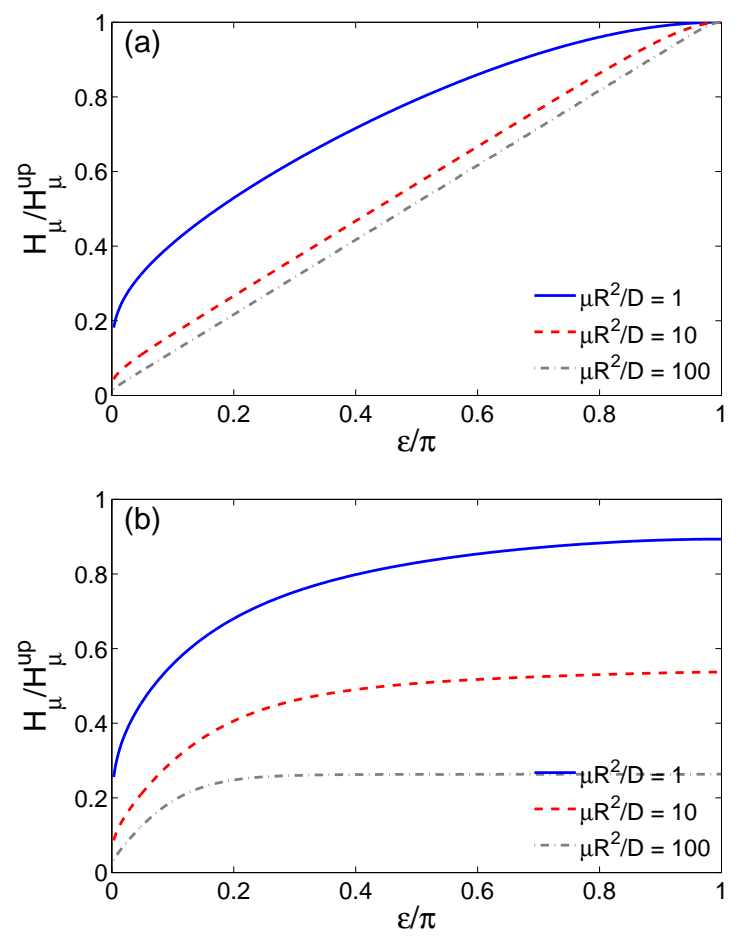

FIG. 7: (Color online) The exit probability $H_{\mu}\left(x_{0}\right)$ (given by Eq. (C10), normalized by its upper bound $H_{\mu}^{\text {up }}\left(x_{0}\right)=$ $U_{2}\left(\left|x_{0}-\partial \Omega\right| \sqrt{\mu / D}\right)$ from Eq. (24), as a function of the normalized perimeter $\varepsilon / \pi$ of the escape arc $(\pi-\varepsilon, \pi+\varepsilon)$, for the disk of radius $R$ at several values of $\mu$. We set $x_{0}=0$ (a) and $x_{0}=-0.5$ (i.e., $r_{0}=0.5$ and $\theta_{0}=\pi$ ) (b).

$x_{0}$, and the rest, one gets

$$
\begin{aligned}
S\left(t ; x_{0}\right) & \leq 1-\frac{\Gamma\left(\frac{d}{2} ;\left|x_{0}-\partial \Omega\right|^{2} /(4 D t)\right)}{\Gamma\left(\frac{d}{2}\right)} \\
& +\left(|\Omega|-\frac{\pi^{\frac{d}{2}}\left|x_{0}-\partial \Omega\right|^{d}}{\Gamma\left(\frac{d}{2}+1\right)}\right) \frac{\exp \left(-\frac{\left|x_{0}-\partial \Omega\right|^{2}}{4 D t}\right)}{(4 \pi D t)^{\frac{d}{2}}}
\end{aligned}
$$

where $\Gamma(\nu, z)$ is the upper incomplete Gamma function, and $|\Omega|$ is the volume of $\Omega$ from which the volume of the ball of radius $\left|x_{0}-\partial \Omega\right|$ is subtracted. The Laplace transform reads

$$
\begin{aligned}
\tilde{S}\left(p ; x_{0}\right) & \leq \frac{1}{p}\left[1-\frac{2^{1-\frac{d}{2}}}{\Gamma\left(\frac{d}{2}\right)} s^{\frac{d}{4}} K_{\frac{d}{2}}(\sqrt{s})\right. \\
& \left.+2^{-\frac{d}{2}}\left(\frac{|\Omega|}{\pi^{\frac{d}{2}} L^{d}}-\frac{1}{\Gamma\left(\frac{d}{2}+1\right)}\right) s^{\frac{d+2}{4}} K_{1-\frac{d}{2}}(\sqrt{s})\right],
\end{aligned}
$$

where $s=\left|x_{0}-\partial \Omega\right|^{2} p / D$, and we used formula 6.453 from 61] for the first Laplace transform involving the incomplete Gamma function. Since $H_{\mu}\left(x_{0}\right)=1-\mu \widetilde{S}\left(\mu ; x_{0}\right)$, this inequality implies a lower bound for the exit proba- 
bility $H_{\mu}\left(x_{0}\right)$. At large $s$, the right-hand side becomes

$$
\begin{aligned}
\tilde{S}\left(p ; x_{0}\right) \lesssim \frac{1}{p}[1 & -\frac{\sqrt{\pi} 2^{-\frac{d+1}{2}}}{\Gamma\left(\frac{d}{2}+1\right)} s^{\frac{d-1}{4}} e^{-\sqrt{s}} \\
& \left.\times\left(d-\left(\frac{|\Omega| \Gamma\left(\frac{d}{2}+1\right)}{\pi^{\frac{d}{2}} L^{d}}-1\right) \sqrt{s}\right)\right] .
\end{aligned}
$$

One can see that the correction to the leading $1 / p$ term vanishes as $e^{-\sqrt{s}}$. However, at large $s$, the correction term becomes positive and thus useless because of the trivial upper bound $\tilde{S}\left(p ; x_{0}\right) \leq 1 / p$. In other words, the upper bound (D3) improves the trivial upper bound $1 / p$ only for moderate values of $p$ (or $s$ ).

We recall that the upper bound (D3) is only applicable for the whole absorbing boundary $(\Gamma=\partial \Omega)$, while its extension to mixed Dirichlet-Neumann boundary condition (with $\Gamma \neq \partial \Omega$ ) is not valid in general. In fact, the domain monotonicity does not hold in general for Neumann heat kernels 60. For instance, in the limit of no escape region, the survival probability is equal to 1 , and the inequality (D1) and its consequence (D3) do not hold. Finding more accurate upper and lower bounds for the Laplace-transformed survival probability for the escape problem presents an important direction for future research.

\section{Appendix E: Marginal probability densities for the FPT and FPP}

In this Appendix, we deduce Eqs. 31, 32). The first equation is simply obtained as

$$
\begin{aligned}
\rho\left(t ; x_{0}\right) & =-\frac{\partial}{\partial t} S\left(t ; x_{0}\right)=-\frac{\partial}{\partial t} \int_{\Omega} d x G_{t}\left(x, x_{0}\right) \\
& =-\int_{\Omega} d x D \Delta G_{t}\left(x, x_{0}\right)=-D \int_{\partial \Omega} d x \frac{\partial}{\partial n} G_{t}\left(x, x_{0}\right) \\
& =\int_{\Gamma} d x q\left(t, x ; x_{0}\right),
\end{aligned}
$$

where we used Eqs. (22, 5), the divergence theorem, the diffusion equation, and boundary conditions.

To deduce Eq. (32), we note that, in analogy to the harmonic measure density, the marginal probability density $\omega_{\mu}(y ; x)$ for the FPP, $y \in \Gamma$, satisfies the following PDE:

$$
\begin{aligned}
{[D \Delta-\mu] \omega_{\mu}(y ; x) } & =0 \quad(x \in \Omega), \\
\omega_{\mu}(y ; x) & =\delta(x-y) \quad(x \in \Gamma), \\
\frac{\partial}{\partial n} \omega_{\mu}(y ; x) & =0 \quad(x \in \partial \Omega \backslash \Gamma) .
\end{aligned}
$$

The density $\omega_{\mu}(y ; x)$ can be expressed through the Laplace-transformed propagator $\tilde{G}_{\mu}\left(x ; x_{0}\right)$ which satisfies

$$
\begin{aligned}
{[D \Delta-\mu] \tilde{G}_{\mu}\left(x ; x_{0}\right) } & =-\delta\left(x-x_{0}\right) \quad(x \in \Omega), \\
\tilde{G}_{\mu}\left(x ; x_{0}\right) & =0 \quad(x \in \Gamma), \\
\frac{\partial}{\partial n} \tilde{G}_{\mu}\left(x ; x_{0}\right) & =0 \quad(x \in \partial \Omega \backslash \Gamma) .
\end{aligned}
$$

Multiplying the first equation in (E2) by $\tilde{G}_{\mu}\left(x ; x_{0}\right)$, the first equation in (E3) by $\omega_{\mu}(y ; x)$, subtracting them and integrating over $x \in \Omega$, one gets

$$
\begin{aligned}
& \omega_{\mu}\left(y ; x_{0}\right) \\
& =D \int_{\Omega} d x\left[\tilde{G}_{\mu}\left(x ; x_{0}\right) \Delta \omega_{\mu}(y ; x)-\omega_{\mu}(y ; x) \Delta \tilde{G}_{\mu}\left(x ; x_{0}\right)\right] \\
& =-D \int_{\partial \Omega} d x\left[\tilde{G}_{\mu}\left(x ; x_{0}\right) \frac{\partial \omega_{\mu}(y ; x)}{\partial n}-\omega_{\mu}(y ; x) \frac{\partial \tilde{G}_{\mu}\left(x ; x_{0}\right)}{\partial n}\right] \\
& =-D \frac{\partial}{\partial n} \tilde{G}_{\mu}\left(x ; x_{0}\right)=\int_{0}^{\infty} d t e^{-\mu t} q\left(t, x ; x_{0}\right)
\end{aligned}
$$

where we used the Green formula and the boundary conditions for both $\tilde{G}_{\mu}\left(x ; x_{0}\right)$ and $\omega_{\mu}(y ; x)$. When $\mu=0$, one retrieves the harmonic measure density, i.e., the probability density of the FPP for immortal walkers.
[1] S. Redner, A Guide to First Passage Processes (Cambridge: Cambridge University press, 2001).

[2] R. Metzler, G. Oshanin, and S. Redner (Eds.) FirstPassage Phenomena and Their Applications (Singapore: World Scientific, 2014).

[3] O. Bénichou and R. Voituriez, "From first-passage times of random walks in confinement to geometry-controlled kinetics", Phys. Rep. 539, 225-284 (2014).

[4] D. Holcman and Z. Schuss, "The Narrow Escape Problem", SIAM Rev. 56, 213-257 (2014).

[5] P Hänggi, P. Talkner, and M. Borkovec, "Reaction-rate theory: fifty years after Kramers", Rev. Mod. Phys. 62, 251 (1990).
[6] P. C. Bressloff and J. M. Newby, "Stochastic models of intracellular transport", Rev. Mod. Phys. 85, 135-196 (2013).

[7] R. Zwanzig and A. Szabo, "Time dependent rate of diffusion-influenced ligand binding to receptors on cell surfaces", Biophys. J. 60, 671-678 (1991).

[8] I. V. Grigoriev, Y. A. Makhnovskii, A. M. Berezhkovskii, and V. Y. Zitserman, "Kinetics of escape through a small hole", J. Chem. Phys. 116, 9574-9577 (2002).

[9] D. Holcman and Z. Schuss, "Escape Through a Small Opening: Receptor Trafficking in a Synaptic Membrane", J. Stat. Phys. 117, 975-1014 (2004).

[10] Z. Schuss, A. Singer, and D. Holcman, "The narrow 
escape problem for diffusion in cellular microdomains", Proc. Nat. Acad. Sci. USA 104, 16098 (2007).

[11] G. M. Viswanathan, S. V. Buldyrev, S. Havlin, M. G. E. da Luz, E. P. Raposok, and H. E. Stanley, "Optimizing the success of random searches", Nature 401, 911-914 (1999).

[12] M. J. Ward and J. B. Keller, "Strong Localized Perturbations of Eigenvalue Problems", SIAM J. Appl. Math. 53, 770-798 (1993).

[13] A. Singer, Z. Schuss, D. Holcman, and R. S. Eisenberg, "Narrow Escape, Part I", J. Stat. Phys. 122, 437-463 (2006).

[14] A. Singer, Z. Schuss, and D. Holcman, "Narrow Escape, Part II The circular disk", J. Stat. Phys. 122, 465-489 (2006).

[15] A. Singer, Z. Schuss, and D. Holcman, "Narrow Escape, Part III Riemann surfaces and non-smooth domains", J. Stat. Phys. 122, 491-509 (2006).

[16] S. Pillay, M. J. Ward, A. Peirce, and T. Kolokolnikov, "An Asymptotic Analysis of the Mean First Passage Time for Narrow Escape Problems: Part I: TwoDimensional Domains", SIAM Multi. Model. Simul. 8, 803-835 (2010).

[17] A. F. Cheviakov, M. J. Ward, and R. Straube, "An Asymptotic Analysis of the Mean First Passage Time for Narrow Escape Problems: Part II: The Sphere", SIAM Multi. Model. Simul. 8, 836-870 (2010).

[18] A. F. Cheviakov, A. S. Reimer, and M. J. Ward, "Mathematical modeling and numerical computation of narrow escape problems", Phys. Rev. E 85, 021131 (2012).

[19] E. Eden, et al. "Proteome Half-Life Dynamics in Living Human Cells", Science 331, 764 (2011).

[20] L. Alvarez, B. M. Friedrich, G. Gompper, and U. B. Kaupp, "The computational sperm cell", Trends Cell Biol. 24, 198-207 (2014).

[21] J. Yang, I. Kupka, Z. Schuss, and D. Holcman, "Search for a small egg by spermatozoa in restricted geometries", J. Math. Biol. 73, 423-446 (2016).

[22] B. Meerson and S. Redner, "Mortality, Redundancy, and Diversity in Stochastic Search", Phys. Rev. Lett. 114, 198101 (2015).

[23] E. Abad, S. B. Yuste, and K. Lindenberg, "Reactionsubdiffusion and reaction-superdiffusion equations for evanescent particles performing continuous-time random walks", Phys. Rev. E 81, 031115 (2010).

[24] E. Abad, S. B. Yuste, and K. Lindenberg, "Survival probability of an immobile target in a sea of evanescent diffusive or subdiffusive traps: A fractional equation approach", Phys. Rev. E 86, 061120 (2012).

[25] E. Abad, S. B. Yuste, and K. Lindenberg, "Evanescent continuous-time random walks", Phys. Rev. E 88, 062110 (2013).

[26] S. B. Yuste, E. Abad, and K. Lindenberg, "Exploration and Trapping of Mortal Random Walkers", Phys. Rev. Lett. 110, 220603 (2013).

[27] B. Meerson, "The number statistics and optimal history of non-equilibrium steady states of mortal diffusing particles", J. Stat. Mech. P05004 (2015).

[28] C. W. Gardiner, Handbook of stochastic methods for physics, chemistry and the natural sciences (Springer: Berlin, 1985).

[29] C. Caginalp and X. Chen "Analytical and Numerical Results for an Escape Problem", Arch. Rational. Mech. Anal. 203, 329-342 (2012).
[30] J.-F. Rupprecht, O. Bénichou, D. S. Grebenkov, and R. Voituriez, "Exit time distribution in spherically symmetric two-dimensional domains", J. Stat. Phys. 158, 192230 (2015).

[31] J. S. Marshall, "Analytical Solutions for an Escape Problem in a Disc with an Arbitrary Distribution of Exit Holes Along Its Boundary", J. Stat. Phys. 165, 920-952 (2016).

[32] A. Godec and R. Metzler, "Universal Proximity Effect in Target Search Kinetics in the Few-Encounter Limit", Phys. Rev. X 6, 041037 (2016).

[33] A. F. Cheviakov and M. Ward, "Optimizing the principal eigenvalue of the Laplacian in a sphere with interior traps", Math. Comp. Modell. 53, 1394-1409 (2011).

[34] S. A. Isaacson and J. Newby, "Uniform asymptotic approximation of diffusion to a small target", Phys. Rev. E 88, 012820 (2013).

[35] P. L. Krapivsky, S. Redner, and E. Ben-Naim, A Kinetic View of Statistical Physics (Cambridge University Press, 2010).

[36] D. S. Grebenkov and G. Oshanin, "Diffusive escape through a narrow opening: new insights into a classic problem" (submitted; available online at ArXiv 1609.00948v1).

[37] R. Milo and R. Philipps, Cell biology by the numbers, (Garland Science and Taylor and Fracis Group, 2015).

[38] K. Z. Pan, T. E. Saunders, I. Flor-Parra, M. Howard, F. Chang, "Cortical regulation of cell size by a sizer cdr2p", eLife 3, e02040 (2014).

[39] F. C. Collins and G. E. Kimball, "Diffusion-controlled reaction rates", J. Coll. Sci. 4, 425 (1949).

[40] H. Sano and M. Tachiya, "Partially diffusion-controlled recombination", J. Chem. Phys. 71, 1276 (1979).

[41] B. Sapoval, "General Formulation of Laplacian Transfer Across Irregular Surfaces", Phys. Rev. Lett. 73, 3314 (1994).

[42] O. Bénichou, M. Moreau, and G. Oshanin, "Kinetics of stochastically gated diffusion-limited reactions and geometry of random walk trajectories", Phys. Rev. E 61, 3388 (2000).

[43] D. S. Grebenkov, Partially Reflected Brownian Motion: A Stochastic Approach to Transport Phenomena, in "Focus on Probability Theory", Ed. L. R. Velle, pp. 135-169 (Nova Science Publishers, 2006).

[44] A. Singer, Z. Schuss, Osipov, and D. Holcman, "Partially Reflected Diffusion", SIAM J. Appl. Math. 68, 844 (2008).

[45] P. C. Bressloff, B. A. Earnshaw, and M. J. Ward, "Diffusion of protein receptors on a cylindrical dendritic membrane with partially absorbing traps", SIAM J. Appl. Math. 68, 1223 (2008).

[46] D. S. Grebenkov, "Analytical representations of the spread harmonic measure density", Phys. Rev. E 91, 052108 (2015).

[47] D. S. Grebenkov, "Searching for partially reactive sites: Analytical results for spherical targets", J. Chem. Phys. 132, 034104 (2010).

[48] D. S. Grebenkov, "Subdiffusion in a bounded domain with a partially absorbing-reflecting boundary", Phys. Rev. E 81, 021128 (2010).

[49] F. Rojo, H. S. Wio, and C. E. Budde, "Narrow-escapetime problem: The imperfect trapping case", Phys. Rev. E 86, 031105 (2012).

[50] O. Bénichou, D. S. Grebenkov, P. Levitz, C. Loverdo, and R. Voituriez, "Optimal Reaction Time for Surface- 
Mediated Diffusion", Phys. Rev. Lett. 105, 150606 (2010).

[51] O. Bénichou, D. S. Grebenkov, P. Levitz, C. Loverdo, and R. Voituriez, "Mean First-Passage Time of SurfaceMediated Diffusion in Spherical Domains", J. Stat. Phys. 142, 657-685 (2011).

[52] F. Rojo and C. E. Budde, "Enhanced diffusion through surface excursion: A master-equation approach to the narrow-escape-time problem", Phys. Rev. E 84, 021117 (2011).

[53] J.-F. Rupprecht, O. Bénichou, D. S. Grebenkov, and R. Voituriez, "Kinetics of Active Surface-Mediated Diffusion in Spherically Symmetric Domains", J. Stat. Phys. 147, 891-918 (2012).

[54] J.-F. Rupprecht, O. Bénichou, D. S. Grebenkov, and R. Voituriez, "Exact mean exit time for surface-mediated diffusion", Phys. Rev. E 86, 041135 (2012).

[55] F. Rojo, C. E. Budde Jr., H. S. Wio, and C. E. Budde, "Enhanced transport through desorption-mediated diffusion", Phys. Rev. E 87, 012115 (2013).
[56] S. B. Yuste and K. Lindenberg, "Subdiffusive target problem: Survival probability", Phys. Rev. E 76, 051114 (2007).

[57] J. Klafter and I. M. Sokolov, First Steps in Random Walks: From Tools to Applications (Oxford University Press, 2011).

[58] J.-P. Bouchaud and A. Georges, "Anomalous diffusion in disordered media: Statistical mechanisms, models and physical applications" Phys. Rep. 195, 127-293 (1990).

[59] R. Metzler, J.-H. Jeon, A. Cherstvy, and E. Barkai, "Anomalous diffusion models and their properties: nonstationarity, non-ergodicity, and ageing at the centenary of single particle tracking", Phys. Chem. Chem. Phys. 16, 24128-24164 (2014).

[60] E. B. Davies, Heat kernels and spectral theory (Cambridge University Press, Cambridge, 1989).

[61] I. S. Gradshteyn and I. M. Ryzhik, Table of Integrals, Series, and Products (Academic Press, 1980). 